\title{
SOME IRREDUCIBLE 2-MODULAR CODES INVARIANT UNDER THE SYMPLECTIC GROUP $S_{6}(2)$
}

\author{
Lucy Chikamai, Jamshid Moori and Bernardo G. Rodrigues \\ University of KwaZulu-Natal and North-West University (Mafikeng), South \\ Africa
}

\begin{abstract}
We examine all non-trivial binary codes and designs obtained from the 2-modular primitive permutation representations of degrees up to 135 of the simple projective special symplectic group $S_{6}(2)$. The submodule lattice of the permutation modules, together with a comprehensive description of each code including the weight enumerator, the automorphism group, and the action of $S_{6}(2)$ is given. By considering the structures of the stabilizers of several codewords we attempt to gain an insight into the nature of some classes of codewords in particular those of minimum weight.
\end{abstract}

\section{INTRODUCTION}

This paper makes an attempt to answer the following general problem: given a prescribed group, determine all invariant $p$-ary codes under the group. This is an enumeration and classification problem which has a merit of its own, but it is also one that lends itself naturally to revealing an interplay between coding theory and modular representation theory. As a by-product one may therefore enumerate and classify all submodules and hence codes invariant under a given group. In [10] and [11] we proposed a novel approach to construct binary codes from the 2-modular primitive representation of a finite group. The said method emerges as a combination of techniques described

2010 Mathematics Subject Classification. 05B05, 20D45, 94 B05.

Key words and phrases. Derived, symmetric and quasi-symmetric designs, selforthogonal designs, codes, optimal linear code, automorphism group, modular representation, symplectic group.

Support of the NRF through incentive and competitive grants are acknowledged by the second author.

This work is based on the research of the third author supported by the National Research Foundation of South Africa (Grant Numbers 84470 and 91495). 
in $[5,6]$ and partially in [20]. In this article, motivated by questions raised in [11] concerning with a certain class of 2 - $(64,28,12)$ designs whose derived designs are not quasi-symmetric, and by the work of Crnković and Mikulić ([13]) we examine binary codes obtained from the permutation modules induced by the action of the simple projective symplectic group $S_{6}(2)$ on the cosets of some of its maximal subgroups. Using a chain of maximal submodules of a permutation module induced by the action of the group $S_{6}(2)$ on various geometrical objects described in [12] as $O_{6}^{-}(2), O_{6}^{+}(2)$, points, $G_{2}(2)$, isotropic planes, isotropic lines, non-isotropic lines and $S_{2}(8)$, we determine all the 2-modular binary linear codes (up to length 135) invariant under the action of the symplectic group $S_{6}(2)$. The submodule lattice of the permutation modules and the weight distribution of the codes obtained from the representations of degrees $28,36,63,120$ and 135 respectively are determined and the incidence relations between the constituents of the representations are given. In addition, an explicit description of the codes is given and where possible using the geometry of the objects described above we provide a geometric interpretation of the nature of the codewords. Moreover, we use the well-known Assmus-Mattson Theorem ([3]) to determine designs which are held by the codewords of given non-trivial weights in the codes. Due to computer time limitations we did not attempt an exhaustive and conclusive study of the remaining representations, namely those of degrees 315,336 and 960. Hence, through computations with MAGma and MEAT-AxE (called within Magma, see [4]), and using the construction prescribed in Lemma 5.1 below we deduce the following main result:

THEOREM 1.1. Let $C$ be a binary linear code of length 28, 36, 63, 120 or 135 invariant under the group $S_{6}(2)$. The following holds:

(i) $\operatorname{Aut}(C)$ is isomorphic to $S_{6}(2), O_{8}^{+}(2), O_{8}^{+}(2): 2$ or $L_{6}(2)$.

(ii) Up to isomorphism there are 214 non-trivial binary codes obtained from the 2-modular representations of $S_{6}(2)$ on these lengths. Of these, 15 are optimal.

The proof of the theorem follows from a series of propositions in Sections 6-10. The paper is organized as follows: after a brief description of our terminology and some background, Sections 3, 4, and 5 give respectively, a description of the simple symplectic group $S_{6}(2)$; the incidence relations among its primitive representations and its 2-modular representations. In Sections $6,7,8,9$, and 10 we present our results.

\section{TERMinOLOGY AND NOTATION}

Our notation for codes and groups will be standard, and it is as in [3] and $[12,23]$. For the structure of groups and their maximal subgroups we follow the Atlas ([12]) notation. The groups $G . H, G: H$, and $G \cdot H$ denote a 
general extension, a split extension and a non-split extension respectively. For a prime $p$, the symbol $p^{m}$ denotes an elementary abelian group of that order. The notation $p_{+}^{1+2 n}$ and $p_{-}^{1+2 n}$ are used for extraspecial groups of order $p^{1+2 n}$. If $p$ is an odd prime, the subscript is + or - according as the group has exponent $p$ or $p^{2}$. For $p=2$ it is + or - according as the central product has an even or odd number of quaternionic factors. If $G$ is a finite group and $\Omega$ is a finite $G$-set, then $F \Omega$ is called a permutation $F G$-module, which has the standard inner product with respect to the basis $\Omega$.

An incidence structure $\mathcal{D}=(\mathcal{P}, \mathcal{B}, \mathcal{I})$, with point set $\mathcal{P}$, block set $\mathcal{B}$ and incidence $\mathcal{I}$ is a $t$ - $(v, k, \lambda)$ design, if $|\mathcal{P}|=v$, every block $B \in \mathcal{B}$ is incident with precisely $k$ points, and every $t$ distinct points are together incident with precisely $\lambda$ blocks. The complementary design of $\mathcal{D}$ is obtained by replacing all blocks of $\mathcal{D}$ by their complements. The design $\mathcal{D}$ is symmetric if it has the same number of points and blocks. The numbers that occur as the size of the intersection of two distinct blocks are the intersection numbers of the design. A $t$ - $(v, k, \lambda)$ design is called self-orthogonal if the intersection numbers have the same parity as the block size. An automorphism of a design $\mathcal{D}$ is a permutation on $\mathcal{P}$ which sends blocks to blocks. The set of all automorphisms of $\mathcal{D}$ forms its full automorphism group denoted by AutD.

The code $C$ of the design $\mathcal{D}$ over the finite field $\mathbb{F}_{p}$ is the space spanned by the incidence vectors of the blocks over $\mathbb{F}_{p}$. The weight enumerator of $C$ is defined as $\sum_{c \in C} x^{\mathrm{wt}(c)}$. The hull of a design $\mathcal{D}$ with code $C$ over the field $F$ is the code obtained by taking the intersection of $C$ and its dual. An $[n, k]$ linear code $C$ is said to be a best known linear $[n, k]$ code if $C$ has the highest minimum weight among all known $[n, k]$ linear codes. An $[n, k]$ linear code $C$ is said to be an optimal linear $[n, k]$ code if the minimum weight of $C$ achieves the theoretical upper bound on the minimum weight of $[n, k]$ linear codes, and near-optimal if its minimum distance is at most 1 less than the largest possible value. A linear $[n, k]$ code is called projective if no two columns of a generator matrix $G$ are linearly dependent, i.e., if the columns of $\mathrm{G}$ are pairwise different points in a projective $(k-1)$-dimensional space. A twoweight code is a code which has exactly two non-zero weights, say $w_{1}$ and $w_{2}$. The dual of a two-weight code belongs to the important family of uniformly packed codes. A code $C$ is self-orthogonal if $C \subseteq C^{\perp}$ and self-dual if equality is attained. The all-one vector will be denoted by $\mathbf{1}$, and is the constant vector of weight the length of the code, and whose coordinate entries consist entirely of 1 's. If $C_{1}$ is an $\left[n_{1}, k_{1}\right]$-code, and $C_{2}$ is an $\left[n_{2}, k_{2}\right]$-code, then we say that $C$ is the direct sum of $C_{1}$ and $C_{2}$ if (up to reordering of coordinates) $C=\left\{(x, y) \mid x \in C_{1}, y \in C_{2}\right\}$. We denote this by $C=C_{1} \oplus C_{2}$. If moreover $C_{1}$ and $C_{2}$ are nonzero, then we say that $C$ decomposes into $C_{1}$ and $C_{2}$. A code $C$ is said to be decomposable if there exist nonzero codes $C_{1}$ and $C_{2}$ such that $C$ decomposes into $C_{1}$ and $C_{2}$. A binary code $C$ is doubly-even if all codewords of $C$ have weight divisible by four. Two linear codes are isomorphic if they 
can be obtained from one another by permuting the coordinate positions. An automorphism of a code is any permutation of the coordinate positions that maps codewords to codewords and will be denoted $\operatorname{Aut}(C)$.

\section{The Group $S_{6}(2)$ AND its PRIMItive PERMUtATion REPRESENTATions}

Below we give a brief overview of the simple symplectic group $G=$ $S_{6}(2)$ (using the Atlas notation) and its maximal subgroups, and primitive permutation representations via the coset action on these subgroups. For a more detailed account on the symplectic groups we refer the reader to $[12$, p. 60], [26, Section 4.5] or [1]. Let $V$ be an $n$-dimensional vector space over a finite field $\mathbb{F}$ and $f$ a non-degenerate alternating bilinear form $f: V \times V \longrightarrow \mathbb{F}$ on V. If $A_{f}$ is the matrix associated with the form $f$, then the group $S p(n, \mathbb{F})$ is defined as $S p(n, \mathbb{F})=\left\{T \in G L(n, \mathbb{F}) \mid T^{t} A_{f} T=A_{f}\right\}$. The factor group of $S p(n, \mathbb{F})$ by its center is called the projective special symplectic group and denoted $P S p(n, q)$ or $S_{n}(q)$ when the group is simple. In terms of matrices the group $S p(n, q)$ is a subgroup of $G L(n, q)$ consisting of the $n \times n$ matrices $P$ satisfying $P^{t} A P=A$, where $A$ is a fixed invertible skew-symmetric matrix. The group $P S p(n, q)$ is simple group for all $n \geq 3$ except when $P S p(4,2) \cong S_{6}$, and when $q=2$ we have that $P S p(n, q) \cong S p(n, q)$ and hence a subgroup of $G L(n, q)$. The group $S_{6}(2)$ is isomorphic to the orthogonal group $O_{7}(2)$, i.e., the group of all $7 \times 7$ matrices preserving a non-singular quadratic form. $S_{6}(2)$ has order 1451520 , and it is its own automorphism group. $G$ acts naturally on the points of the projective geometry $P G(5,2)$. It is known that $P G(5,2)$ has 63 points and 651 lines and in addition 315 totally isotropic lines, and 135 totally isotropic planes. $G$ has 8 primitive permutation representations of degrees $28,36,63,120,135,315,336$ and 960 respectively (see [12] or [1]). These representations are shown in Table 1: The first column gives the ordering of the primitive representations as given by Magma (or the Atlas) and as used in our computations; the second gives the degree (the number of cosets of the point stabilizer); the third gives the maximal subgroups; the fourth gives the number of orbits, and the remaining column gives the length of the orbits of the point stabilizer.

TABLE 1. Maximal subgroups of $S_{6}(2)$.

\begin{tabular}{|l|l|l|l|l|}
\hline no. & degree & Max subgroup & \# of orbits & orbit length \\
\hline 1 & 28 & $U_{4}(2): 2$ & 2 & 1,27 \\
2 & 36 & $S_{8}$ & 2 & 1,35 \\
3 & 63 & $2^{5}: S_{6}$ & 3 & $1,30,32$ \\
4 & 120 & $U_{3}(3): 2$ & 3 & $1,56,63$ \\
5 & 135 & $2^{6}: L_{3}(2)$ & 4 & $1,14,56,64$ \\
6 & 315 & $2 \cdot\left[2^{6}\right]:\left(S_{3} \times S_{3}\right)$ & 5 & $1,18,24,128,144$ \\
7 & 336 & $S_{3} \times S_{6}$ & 5 & $1,20,45,90,180$ \\
8 & 960 & $L_{2}(8): 3$ & 6 & $1,56,63,84,252,504$ \\
\hline
\end{tabular}


We summarize the information obtained from the group and find notations for the objects which are permuted in each of its primitive permutation representations. The primitive representations may also be described (often is several ways, see for example the Atlas [12]) in terms of the action of $G$ on various sets of geometrical objects: we shall use the notations $g(m)(m=28,36,63,120,135,315,336$ and 960$)$ to denote these sets. We will use names for all objects in terms of their symplectic specifications from [12], namely $O_{6}^{-}(2), O_{6}^{+}(2)$, points, $G_{2}(2)$, isotropic planes, isotropic lines, non-isotropic lines and $S_{2}(8)$.

\section{INCIDENCE RELATIONS}

The action of a group fixing an element of $g(m)$ may be transitive on the elements of $g(n)$ or may split these elements into several orbits or into two orbits if $m \neq n$, of which one has size one if $m=n$. The rows and columns of Table 2 represent the intersection of the objects being permuted as named above. If we denote the entries in Table 2 by $a_{m n}$, then the entry $a_{33}$ corresponds the action of $2^{5}: S_{6}$ on its cosets in $S_{6}(2)$ which produces three orbits of lengths 1,30 and 32 respectively. However the entry $a_{73}$ indicates that there are three orbits of an intransitive action of an $S_{3} \times S_{6}$ on $2^{5}: S_{6}$, of lengths 3,15 and 45 and the entry $a_{57}$ indicates a transitive action of an $2^{6}: L_{3}(2)$ on $S_{3} \times S_{6}$.

TABLE 2. Orbits of primitive permutation representations of $S_{6}(2)$

\begin{tabular}{|c|c|c|c|c|c|c|c|c|}
\hline & 28 & 36 & 63 & 120 & 135 & 315 & 336 & 960 \\
\hline \hline 28 & $1-27$ & 36 & $27-36$ & 120 & 135 & $45-270$ & $120-216$ & 960 \\
\hline 36 & 28 & $1-35$ & $28-35$ & 120 & $30-105$ & $105-210$ & $56-280$ & 960 \\
\hline 63 & $12-16$ & $16-20$ & $1-30-32$ & 120 & 15120 & $15-60-240$ & $16-80-240$ & 960 \\
\hline 120 & 28 & 36 & 63 & $1-56-63$ & $63-72$ & $63-252$ & 336 & $288-672$ \\
\hline 135 & 28 & $8-28$ & $7-56$ & $56-64$ & $1-14-56-64$ & $7-84-224$ & $112-224$ & $64-896$ \\
\hline 315 & $4-24$ & $12-24$ & $3-12-48$ & $24-96$ & $3-36-96$ & $1-18-24-128-144$ & $16-48-128-144$ & $192-768$ \\
\hline 336 & $10-18$ & $6-30$ & $3-15-45$ & 120 & $45-90$ & $15-45-120-135$ & $1-20-45-90-180$ & $240-720$ \\
\hline 960 & 28 & 36 & 63 & $36-84$ & $9-126$ & $63-252$ & $84-252$ & $1-56-63-84-252-504$ \\
\hline
\end{tabular}

We have considered the 2-modular representations of degrees 28, 36, 63, 120 and 135 and omitted those of degrees 315,336 and 960 respectively due to computer time limitations.

\section{The 2-Modular Representations of $S_{6}(2)$ AS Binary CODES}

Each conjugacy class of maximal subgroups of $S_{6}(2)$ generates a permutation module over $\mathbb{F}_{2}$. We shall consider these $\mathbb{F}_{2}$-modules, and their invariant submodules under the action of $G$. Starting with the permutation module we recursively find maximal submodules filtering any isomorphic copies until we obtain an irreducible module. Each maximal submodule constitutes in turn the binary code that is invariant under $S_{6}(2)$. After eliminating isomorphic 
copies, we obtain a lattice of submodules. We shall consider these $\mathbb{F}_{2^{-}}$ modules, and a chain of all their invariant maximal submodules under the action of $S_{6}(2)$. In this way, we classify and enumerate all submodules, hence codes invariant under $S_{6}(2)$. Taking the submodules as the working modules, the corresponding maximal submodules are found recursively. The recursion terminates as soon as we reach an irreducible maximal submodule or a maximal submodule of dimension 1 . In so doing we determine all codes associated with the permutation module of a given dimension and invariant under the group. Our construction is based on a method outlined in [10] which is made explicit by the following lemma whose proof we present for completeness.

LEMMA 5.1 ([9,24]). Let $G$ be a finite group and $\Omega$ a finite $G$-set. Then the $\mathbb{F}_{2} G$-submodules of $\mathbb{F}_{2} \Omega$ are precisely the $G$-invariant codes (i.e., $G$-invariant subspaces of $\mathbb{F}_{2} \Omega$ ).

Proof. Let $G$ be a finite permutation group acting on a finite set $\Omega$ in the usual way. Let $V=\mathbb{F}_{2} \Omega$ be the $\mathbb{F}_{2}$-vector space with basis the elements of $\Omega$. Let $\rho: G \longrightarrow G L(V)$ be a representation of $G$ given by

$$
\rho(g)(x)=g(x) \text { for all } g \in G \text { and } x \in V \text {. }
$$

We can consider $V$ as the $\mathbb{F}_{2} G$-module obtained from $\rho$. Let $\mathcal{S}$ be an $\mathbb{F}_{2} G$ submodule of the permutation module $V$. Then since $\mathcal{S}$ is a $G$-invariant code we have

$$
\left(\sum_{g \in G} \alpha_{g} g\right) \cdot S \in \mathcal{S} \text { for all } \sum_{g \in G} \alpha_{g} g \in \mathbb{F}_{2} G \text { and } S \in \mathcal{S} .
$$

In particular,

$$
g \cdot S \in \mathcal{S} \text { for all } g \in G \text { and } S \in \mathcal{S} .
$$

Thus, for all $g \in G$ and $S \in \mathcal{S}$ we obtain $\rho(g)(S) \in \mathcal{S}$ or $g(S) \in \mathcal{S}$ and so $\mathcal{S}$ is $G$-invariant. Conversely, if $\mathcal{S}$ is $G$-invariant, then for all $g \in G$ and $S \in \mathcal{S}$ we have $\rho(g)(S) \in \mathcal{S}$. Therefore for scalars $\alpha_{g} \in \mathbb{F}_{2}$ we have

$$
\sum_{g \in G} \alpha_{g} \rho(g)(S) \in \mathcal{S}
$$

by linearity. This implies that

$$
\left(\sum_{g \in G} \alpha_{g} g\right) \cdot S \in \mathcal{S}
$$

The construction outlined in Lemma 5.1 requires that we find all submodules of the given permutation module. For this we decompose the permutation module into submodules. These constitutes the building blocks 
for the construction of a lattice of submodules where possible, thus attaining an answer to the enumeration problem. With the characterization of these codes we respond to the problem of classification of the codes.

Considering $G$ to be the simple symplectic group $S_{6}(2)$, the sections that follow present the calculations on the $G$-invariant modules by making use of Lemma 5.1. The vectors in each submodule form a code, over $\mathbb{F}_{2}$, whose length is the dimension of the permutation module and whose dimension is the dimension of the submodule. The weight enumerators of the submodules are therefore also the weight enumerators of these codes which are invariant under the action of $S_{6}(2)$.

\section{28-DIMENSIONAL REPRESENTATION}

In its representation on a set $\Omega=\{1,2, \ldots, 28\}$ the group $S_{6}(2)$ has for point stabilizer $U_{4}(2): 2$ which has two orbits of lengths 1 and 27 respectively. Using the Atlas [12], we notice that the constituents being permuted by the group are the 28 symbols (copies of $O_{6}^{-}(2)$ ) of the set $\Omega$. The permutation module splits into three absolutely irreducible constituents of dimension 1,6 and 14 with multiplicities 2, 2 and 1 respectively. There is only one irreducible submodule of dimension 1. Moreover, the permutation module has only one maximal submodule of dimension 27. In fact the permutation module has only one composition series, namely: $\mathbb{F}_{2} \Omega=28 \supseteq 27 \supseteq 21 \supseteq 7 \supseteq 1$. These codes and their duals will be denoted $C_{28, i}$ and $C_{28, i}{ }^{\perp}$ and their properties are discussed below in Proposition 6.1.

Figure 1. Submodule lattice of the 28-dimensional permutation module

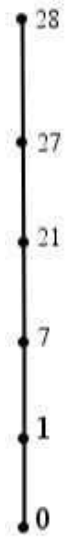


TABLE 3. Weight distribution of the codes from the 28dimensional representation.

\begin{tabular}{|c|c|c|c|c|c|c|c|c|c|c|c|c|c|}
\hline Name & $\operatorname{dim} \theta$ & 04 & 6 & 8 & 10 & 12 & 14 & 16 & 18 & 20 & 22 & 24 & 28 \\
\hline & 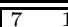 & $\overline{1}$ & & & & $\overline{\overline{63}}$ & & $\overline{\overline{63}}$ & & & & & $\overline{\overline{1}}$ \\
\hline$C_{28, \perp}$ & 21 & 1315 & 6048 & 47817 & 206976 & 472059 & 630720 & 472059 & 206976 & 47817 & 6048 & 315 & 1 \\
\hline
\end{tabular}

Proposition 6.1. The code $C_{28,1}$ is self-orthogonal and doubly-even, with minimum weight 12. It is a $[28,7,12]_{2}$ code, and its dual $C_{28,1}{ }^{\perp}$ is $a[28,21,4]_{2}$ singly even code. $\mathbf{1} \in C_{28,1}$ and $\mathbf{1} \in C_{28,1}{ }^{\perp}$. Moreover, $C_{28,1}$ and $C_{28,1}{ }^{\perp}$ are optimal codes that are generated by their minimum weight codewords, and $\operatorname{Aut}\left(C_{28,1}\right) \cong S_{6}(2)$

Proof. See [11, Proposition 8.1](iii) or [14].

REMARK 6.2. The code $C_{28,1}$ was discussed in [11] in connection with codes obtained from the 2-modular representation of $A_{8}$. It is worth pointing out that $A_{8} \cong O_{6}^{-}(2) \leq S_{6}(2)$. From the Atlas ([12]) we infer that the words of minimum weight represent the points of the projective space $P G(5,2)$ or the isotropic points in the orthogonal space of dimension 7; illustrating yet again the isomorphism between $S_{6}(2)$ and $O_{7}^{+}(2)$. The stabilizer of a point in this action is a group isomorphic to the group $U_{4}(2): 2$. The image under $S_{6}(2)$ of the codewords of minimum weight form a $2-(28,12,11)$ design on which $S_{6}(2)$ acts primitively. Moreover, the codewords of minimum weight in $C_{28,1}{ }^{\perp}$ represent the isotropic lines. The stabilizer of an isotropic line is a group isomorphic to $\left(2^{1+4} \times 2^{2}\right):\left(S_{3} \times S_{3}\right)$. Their minimum words represent the blocks of a $2-(28,4,5)$ design with 315 blocks isomorphic to the well-known Hölz design.

\section{36-DIMENSIONAL REPRESENTATION}

Notice from Table 1 (see also Table 2) that there is a single class of maximal subgroups of $S_{6}(2)$ of index 36 when $G$ acts on the cosets of $S_{8}$. Under this action $S_{8}$ has two orbits, one of length 1 and another of length 35 respectively and we get a permutation representation of degree 36 . Hence we form a permutation module of dimension 36 invariant under $G$. By [12] the elements being permuted by $G$ are copies of $S_{8}$. The permutation module splits into 4 absolutely irreducible constituents of dimensions 1, 6, 8 and 14 with multiplicities of 2, 2, 1 and 1 respectively. There is only one irreducible submodule of dimension 1 . Moreover the permutation module has only one maximal submodule of dimension 35. Now, from the 35-dimensional module we get one maximal submodule of dimension 29. From the 29-dimensional module we get two maximal submodules of dimensions 15 and 21 respectively. These two modules contains one maximal submodule each of dimension 7 which has in turn one irreducible maximal submodule of dimension 1. 
We thus find that the permutation module has submodules of dimensions $35,29,21,15,7$ and 1 and hence obtain 4 non trivial codes of dimensions $7,15,21$ and 29. The lattice of the submodules is as shown in Figure 2 and the weight distribution given in Table 4 .

Figure 2. Submodule lattice of the 36 dimensional permutation module

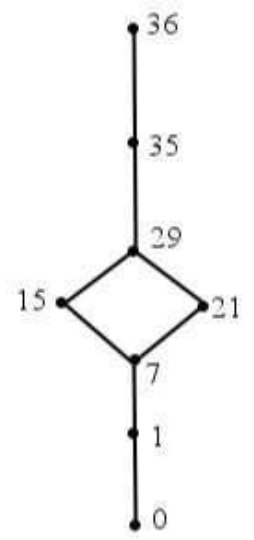

TABLE 4. The weight distribution of the codes from a 36dimensional representation.

\begin{tabular}{|c|c|c|c|c|c|c|c|c|c|c|}
\hline Name & $\operatorname{dim}$ & $\begin{array}{l}0 \\
36\end{array}$ & $\begin{array}{l}4 \\
32\end{array}$ & $\begin{array}{l}6 \\
30\end{array}$ & $\begin{array}{l}8 \\
28\end{array}$ & $\begin{array}{l}10 \\
26\end{array}$ & $\begin{array}{l}12 \\
24\end{array}$ & $\begin{array}{l}14 \\
22\end{array}$ & $\begin{array}{l}16 \\
20\end{array}$ & 18 \\
\hline$C_{36,1}$ & 7 & 1 & & & & & & & 63 & \\
\hline$C_{36,2}$ & 15 & 1 & & & 135 & & 945 & 4320 & 7623 & 6720 \\
\hline$C_{36,2 \perp}$ & 21 & 1 & & 336 & 945 & 16128 & 78435 & 229680 & 440811 & 564480 \\
\hline$C_{36,1 \perp}^{50,2-}$ & 29 & 1 & 945 & 30576 & 471420 & 3977568 & 19541340 & 59348880 & 114138486 & 141852480 \\
\hline
\end{tabular}

Notice that all the codes are self-complementary and that $C_{36,1}$ is a subcode of all the codes. In addition $C_{36,1^{\perp}}$ contains all of them. The containments amongst the codes is given in Proposition 7.1 and a detailed description of them in Proposition 7.2.

Proposition 7.1.

(i) $C_{36,1} \subset C_{36,2} \subset C_{36,1^{\perp}}$

(ii) $C_{36,2^{\perp}} \subset C_{36,1^{\perp}}$

Proposition 7.2. (i) The code $C_{36,1}$ is self-orthogonal and doublyeven, with minimum weight 16 . It is a $[36,7,16]_{2}$ code, and its dual $C_{36,1}{ }^{\perp}$ is a $[36,29,4]_{2}$ singly even code. $\mathbf{1} \in C_{36,1}$ and $\mathbf{1} \in C_{36,1}{ }^{\perp}$ with $C_{36,1}, C_{36,1} \perp$ optimal codes that are generated by their minimum weight codewords. Moreover, $\operatorname{Aut}\left(C_{36,1}\right) \cong S_{6}(2)$ and $S_{6}(2)$ acts irreducibly on $C_{36,1}$. 
(ii) The code $C_{36,2}$ is singly-even with minimum weight 8 . It is a $[36,15,8]_{2}$ code, and its dual $C_{36,2}{ }^{\perp}$ is a $[36,21,6]_{2}$ code. Moreover $\operatorname{Aut}\left(C_{36,2}\right) \cong S_{6}(2)$. $C_{36,2}$ is a distance 2 from optimal while $C_{36,2}{ }^{\perp}$ is near-optimal. Both codes are generated by their minimum weight codewords.

Proof. (i) $S_{6}(2)$ acts 2-transitively on the set of co-ordinates of $C_{36, i}$ for $1 \leq i \leq 2$, and so we have that the support of a codeword of any fixed non-zero weight in $C_{36, i}$ yields a 2-design. In particular we can show that the support of the minimum weight codewords yield a $2-(36,16,12)$ design with 63 blocks. We denote this design $\mathcal{D}_{w_{16}}$ and can show that the blocks of $\mathcal{D}_{w_{16}}$ meet in 6 or 8 points. Thus $\mathcal{D}_{w_{16}}$ is a quasi-symmetric design. Now, since in $\mathcal{D}_{w_{16}}$ we have that $\left|B_{i} \cap B_{j}\right|=\{6,8\} \equiv 0(\bmod 2)$, with $B_{i}$ and $B_{j}$ two distinct blocks and $k=16 \equiv 0(\bmod 2)$, we have a self-orthogonal design. Thus, the point block incidence matrix of $\mathcal{D}_{w_{16}}$ spans a self-orthogonal code of length 36 , which we denote $C_{36,1}$. Since the block size of $\mathcal{D}_{w_{16}}$ is even we have that $\mathbf{1} \in C_{36,1}{ }^{\perp}$. Since the code is spanned by its minimumweight codewords whose weights are divisible by four, it is doubly-even. Notice from Table 4 that the weight distribution of $C_{36,1}$ is $A_{0}=A_{36}=1$ and $A_{16}=A_{20}=63$ and the minimum-weight codewords are the incidence vectors of the blocks of the design and those of weight 20 are the incidence vectors of the blocks of the complementary design. Now, from [21, Theorem 1] or $\left[13\right.$, Table 4] we have that $\operatorname{Aut}\left(\mathcal{D}_{w_{16}}\right) \cong S_{6}(2)$. But $\operatorname{Aut}\left(\mathcal{D}_{w_{16}}\right) \subseteq \operatorname{Aut}\left(C_{36,1}\right)$ and $\left|\operatorname{Aut}\left(C_{36,1}\right)\right|=\left|S_{6}(2)\right|$, and so the result follows. Furthermore, since $r=28 \neq 2 \lambda=24$ and $C_{36,1}{ }^{\perp} \neq 0$ we have that the minimum-weight of $C_{36,1}{ }^{\perp}$ is at least 4 . From the 2-modular character table of $S_{6}(2)$ (see [19]) we have that 7 is the smallest dimension for any non-trivial irreducible $\mathbb{F}_{2}$-module invariant under $S_{6}(2)$. Irreducibility now follows easily by using the weight enumerator of the code. The optimality of the codes was found using Magma and verified in the online tables of optimal codes, see [16]. This also follows if we regard $\mathcal{D}_{w_{16}}$ as the residual design of a symmetric difference property design, (see [21] for more details). In this way, we obtain a code meeting the Grey-Rankin bound with parameters $(36,128,16)$ and of minimum possible 2-rank 7, which is optimal. Finally, since $\mathbf{1} \in C_{36,1}$ it follows that the code of the complementary $2-(36,20,19)$ design is $C_{36,1}$.

(ii) Similarly the support of the codewords of weight 8 in $C_{36,2}$ holds a 2 $(36,8,6)$ design $D_{w_{6}}$ with 135 blocks. The row vectors of the point block incidence matrix of this design generate the code of length 36 denoted $C_{36,2}$ with $\operatorname{Aut}\left(C_{36,2}\right) \cong S_{6}(2)$. Form the online tables of optimal codes we can easily verify that $C_{36,2}$ is a distance 2 from optimal, while $C_{36,2}{ }^{\perp}$ is near-optimal.

As an immediate consequence of Proposition 7.2 and the results given in Section 7 we deduce the following result. 
COROLlary 7.3. Up to isomorphism there are exactly 4 non-trivial codes of length 36 invariant under $G$. There is no $G$-invariant self-dual code $C$ of length 36 .

REMARK 7.4. The attentive reader will observe the connection between the designs and codes obtained from the permutation module of dimension 28 discussed in Section 6 and those given in Section 7. It should also become evident to the reader that since $A_{8} \leq S_{6}(2)$ this connection is natural. In what follows we attempt to outline this interplay between the codes and designs in a more detailed manner:

(i) The designs $2-(28,12,11)$ given in Section 6 and $\mathcal{D}_{w_{16}}$ are respectively the derived and the residual designs of a $2-(64,28,12)$ design and they are part of a infinite family of quasi-symmetric designs constructed from the symplectic group $S_{2 m}(2)$ and quadratic forms, see $[13,21]$. These designs are on $v=$ $2^{2 m-1} \pm 2^{m-1}$ points depending on whether we consider hyperbolic or elliptic quadratic forms. Note that the codes $C_{28,1}$ and $C_{36,1}$ are isomorphic as $\mathbb{F}_{2^{-}}$ modules. In Proposition 7.2 we saw that all codes have $S_{6}(2)$ as their full automorphism group. After a careful examination of Table 4 we deduce that the non-zero weight codewords of the codes $C_{36, i} 1 \leq i \leq 3$ are single orbits and are stabilized by maximal subgroups of the automorphism groups. We consider the action of $\operatorname{Aut}(C)=S_{6}(2)$ on the codewords of minimum weight to describe the structure of the stabilizers and form 2-designs which are invariant under $S_{6}(2)$. Using this information we describe the nature of the codewords of minimum weight. For this, let $w_{m}$ denote a codeword of a nonzero weight $m$ in $C=C_{36, i}$. If we take $m \in\{16,8,6\}$ for $C_{36, i}, 1 \leq i \leq 3$, respectively then from Table 4 we see that $w_{m}{ }^{S_{6}(2)}$ forms a single orbit and so $S_{6}(2)$ is transitive on the code coordinates. Now, using Table 4 and the orbit stabilizer theorem we have $\left[S_{6}(2):\left(S_{6}(2)_{w_{m}}\right)\right] \in\{63,135,336\}$. This implies that $\left(S_{6}(2)\right)_{w_{m}} \in$ $\left\{2^{5}: S_{6}, 2^{6}: L_{3}(2), S_{3} \times S_{6}\right\}$. Since $S_{6}(2)$ is transitive on code coordinates, the support of the codewords of the given weights yield the designs $\mathcal{D}_{w_{m}}$. These are in fact $2-(36,16,12), 2-(36,8,6)$, and $2-(36,6,8)$ designs. The number of blocks in the designs equal the indices of $\left(S_{6}(2)\right)_{w_{m}}$ in $S_{6}(2)$. Hence, $S_{6}(2)$ acts primitively on $\mathcal{D}_{w_{m}}$.

(ii) Using the above information we can give a geometric interpretation of codewords of minimum weight. From [12], note that codewords of weight 16 in $C_{36,1}$ represent the points of the projective space $P G(5,2)$ and the stabilizer of a point is a group isomorphic to $2^{5}: S_{6}$. The codewords of weight 16 are also the blocks of the design $D_{w_{16}}$ described earlier. The codewords of weight 8 in $C_{36,2}$ represent the isotropic planes, and the stabilizer of an isotropic plane is isomorphic to a group of shape $2^{6}: L_{3}(2)$. Moreover, the image of their support under the action of the group form the blocks of a 2-(36, 8, 6) design. Similarly the codewords of weight 6 in $C_{36,2}{ }^{\perp}$ represent non-isotropic lines, and the stabilizer of a non-isotropic line is a group isomorphic to $S_{6} \times S_{3}$. 
Furthermore, the support of the codewords of minimum weight 6 form the blocks of a $2-(36,6,8)$ design.

(iii) The designs with parameters $2-(28,10,40), 2-(36,12,33)$, and 2 $(36,6,8)$ where first obtained in [13]. The authors queried in that paper whether or not such designs were known to exist. The $2-(28,10,40)$ design is constructed using the codewords of weight 10 in $C_{28,1}{ }^{\perp}$. From the fixed points of a Sylow 3-subgroup of $2^{4}: S_{5}$ one can construct the 2- $(28,10,40)$ design. Furthermore, the $2-(36,6,8)$ design is defined by the ovals of the $2-(36,8,6)$ design, see [13]. Here, we used the codes and the supports of codewords of given non-zero weight to show yet another way of constructing such designs and also provide geometric interconnections which uncover the interplay between coding theory and design theory via modular representation theory.

\section{63-DIMENSIONAL REPRESENTATION}

It follows from Section 3 and it can also be deduced from Tables 1 and 2 that $S_{6}(2)$ acts primitively as rank-3 group of degree 63 on the points of the projective geometry $P G(5,2)$. The stabilizer of a point is a group isomorphic to $2^{5}: S_{6}$ with orbits of lengths 1,30 and 32 respectively. It is well known that such an action defines a strongly regular $(63,30,13,15)$ graph. We denote this graph $\Gamma$ and remark that its complement is a strongly regular $(63,32,16,16)$ graph. Since $\Gamma$ is a graph that appears in a partition of the symplectic graph $\mathcal{S}_{6}^{+}(2)$, it follows from [22, Theorem 5.3$]$ that $\Gamma$ possesses the triangle property and as such it is uniquely determined by its parameters and by the minimality of its 2-rank. For completeness, we give an overview of the symmplectic graph. Let $\mathcal{A}$ be a $2 n \times 2 n$ nonsingular alternate matrix over $\mathbb{F}_{q}$, the symplectic graph relative to $\mathcal{A}$ over $\mathbb{F}_{q}$ is the graph with the set of one-dimensional subspaces of $\mathbb{F}_{q}{ }^{(2 n)}$ as its vertex set and with adjacency defined by $[u] \sim[v]$ if and only if $u \mathcal{A}^{t} v \neq 0$ for any $u \neq 0$ and $v \neq 0 \in \mathbb{F}_{q}{ }^{(2 n)}$, where $[u]$ and $[v]$ are one-dimensional subspaces of $\mathbb{F}_{q}{ }^{(2 n)}$, and $[u] \sim[v]$ means that $[u]$ and $[v]$ are adjacent.

Using this action a permutation module of dimension 63 is formed. Moreover, from [12] we can identify the elements being permuted by the group as being the points of the projective geometry. The permutation module splits into four absolutely irreducible constituents of dimension 1, 6,8 and 14 with multiplicities of $3,4,1$ and 2 respectively. We found that there are two irreducible submodules of dimension 1 and 6 both absolutely irreducible. Moreover, the 63-dimensional permutation module has two maximal submodules of dimension 57 and 62 . Now, from the 57dimensional module we get four non-isomorphic maximal submodules of dimension $43,56,56,56$, and from the 62 -dimensional module we get one 
maximal submodule of dimension 56 which is isomorphic to the third 56dimensional submodule. From the 43-dimensional submodule we get three non-isomorphic maximal submodules each of dimension 42. From each of the 56-dimensional submodules we get two maximal submodules, one of dimension 42 and the other of dimension 55. The three 55-dimensional submodules are all isomorphic. We find that the three 42-dimensional submodules are all non-isomorphic, although being each isomorphic to a 42 dimensional submodule obtained from the 43-dimensional submodule. We now have four maximal submodules each of dimension 42,42,42 and 55 . From each of these, we get one maximal submodule of dimension 41 and in addition we get another maximal submodule of dimension 36 from the third 42-dimensional submodule. Analogous to the representations of degrees 28 and 36, using Meat-Axe we worked recursively through the chain of submodules of the permutation module and filtered out isomorphic copies of maximal submodules. In doing so, we determined a total of 27 submodules of dimensions $1,6,7,7,7,8,20,21,21,21,22,27,28,35,36,41,42,42,42,43$, $55,56,56,56,57,62$ and 63 respectively. The lattice of submodules is shown in Figure 3. We obtain a total of 24 non-trivial binary codes of dimensions $6,7,7,7,8,20,21,21,21,22,27,28,35,36,41,42,42,42,43,55,56,56,56$ and 57 respectively. These codes and their duals will be denoted $C_{63, i}$ and $C_{63, i}{ }^{\perp}$, with $1 \leq i \leq 12$ in an increasing order of their dimension. The weight distributions of the codes and those of their duals are given in Tables 5 and 7 respectively. We note that in Table 7 we give only a partial listing of the weight distribution of the duals, since the weights are too large.

Figure 3. Submodule lattice of the 63-dimensional permutation module

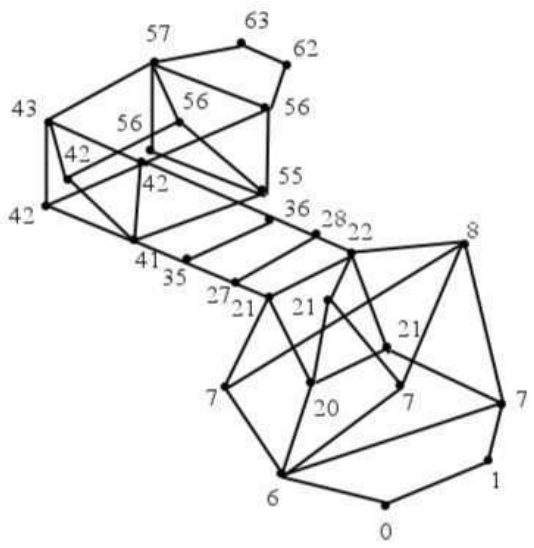


TABLE 5. The weight distribution of the codes from a 63dimensional representation.

\begin{tabular}{|c|c|c|c|c|c|c|c|c|c|c|c|c|}
\hline Name & $\operatorname{dim}$ & $\begin{array}{lll}0 & 11 \\
\end{array}$ & 12 & 15 & 16 & 19 & 20 & 23 & 24 & 27 & 28 & 31 \\
\hline$\overline{C_{63,1}}$ & 6 & $\overline{1}$ & & & & & & & & & & \\
\hline$C_{63,2}$ & 7 & 1 & & & & & & & & & 36 & \\
\hline$C_{63,3}$ & 7 & 1 & & & & & & & & 28 & & \\
\hline$C_{63,4}$ & 7 & 1 & & & & & & & & & & 63 \\
\hline$C_{63,5}$ & 8 & 1 & & & & & & & & 28 & 36 & 63 \\
\hline$C_{63,6}$ & 20 & 1 & & & 945 & & & & 91560 & & 248832 & \\
\hline$C_{63,7}$ & 21 & 1 & & & 1953 & & & & 182280 & & 499968 & \\
\hline$C_{63,8}$ & 21 & 1 & & 336 & 945 & & & 54432 & 91560 & 195328 & 248832 & 455616 \\
\hline$C_{63,9}$ & 21 & 1 & & 315 & 945 & & & 54936 & 91560 & 193536 & 248832 & 458451 \\
\hline$C_{63,10}$ & 22 & 1 & & 651 & 1953 & & & 109368 & 182280 & 388864 & 499968 & 914067 \\
\hline$C_{63,11}$ & 27 & 1 & 1638 & & 20097 & & 749826 & & 9274440 & & 36318492 & \\
\hline$C_{63,12}$ & 28 & 1378 & 1638 & 6699 & 20097 & 340830 & 749826 & 5564664 & 9274440 & 28247716 & 36318492 & 53692947 \\
\hline
\end{tabular}

TABle 6. Table 5 continued.

\begin{tabular}{|c|c|c|c|c|c|c|c|c|c|c|c|c|}
\hline $\operatorname{dim}$ & 32 & 35 & 36 & 39 & 40 & 43 & 44 & 47 & 48 & 51 & 52 & 63 \\
\hline$C_{63,1}$ & 63 & & & & & & & & & & & \\
\hline$C_{63,2}$ & 63 & & 28 & & & & & & & & & \\
\hline$C_{63,3}$ & 63 & 36 & & & & & & & & & & \\
\hline$C_{63,4}$ & 63 & & & & & & & & & & & 1 \\
\hline$C_{63,5}$ & 63 & 36 & 28 & & & & & & & & & 1 \\
\hline$C_{63,6}$ & 458451 & & 193536 & & 54936 & & & & 315 & & & \\
\hline$C_{63,7}$ & 914067 & & 388864 & & 109368 & & & & 651 & & & \\
\hline$C_{63,8}$ & 458451 & 251136 & 193536 & 90720 & 54936 & & & 1008 & 315 & & & \\
\hline$C_{63,9}$ & 458451 & 248832 & 193536 & 91560 & 54936 & & & 945 & 315 & & & 1 \\
\hline$C_{63,10}$ & 914067 & 499968 & 388864 & 182280 & 109368 & & & 1953 & 651 & & & 1 \\
\hline$C_{63,11}$ & 53692947 & & 28247716 & & 5564664 & & 340830 & & 6699 & & 378 & \\
\hline$C_{63,12}$ & 53692947 & 36318492 & 28247716 & 9274440 & 5564664 & 749826 & 340830 & 20097 & 6699 & 1638 & 378 & 1 \\
\hline
\end{tabular}

TABLE 7. Partial listing of the weight distribution of the dual of codes of length 63

\begin{tabular}{|c|c|c|c|c|c|c|c|c|c|c|}
\hline Name & $\operatorname{dim}$ & 03 & 4 & 5 & 6 & 7 & 8 & $\overline{9}$ & 10 & 11 \\
\hline$\overline{C_{6312 \perp}}$ & 35 & $\overline{1}$ & & & & & 945 & & & \\
\hline$C_{63,11 \perp}^{63,12 \perp}$ & 36 & 1 & & & & 135 & 945 & & & 6048 \\
\hline$C_{63,10} \perp$ & 41 & 1 & & & & & 9765 & & & \\
\hline$C_{63,9 \perp} \perp$ & 42 & 1 & & & & & 11781 & & 60480 & \\
\hline$C_{63,8} \perp$ & 42 & 1 & & & & 288 & 9765 & 11200 & & 294336 \\
\hline$C_{63,7^{\perp}}$ & 42 & 1 & & & & 1395 & 9765 & & & 328104 \\
\hline$C_{63,6} \perp$ & 43 & 1 & & & & 1683 & 11781 & 11200 & 60480 & 622440 \\
\hline$C_{63,5} \perp$ & 55 & 1 & 4725 & & 531048 & & 30252537 & & 998505144 & \\
\hline$C_{63,4^{\perp}}$ & 56 & 1 & 9765 & & 1057224 & & 60544953 & & 1996794072 & \\
\hline$C_{63,3} \perp$ & 56 & 1336 & 4725 & 54432 & 531048 & 4327488 & 30252537 & 184868320 & 998505144 & 4811041872 \\
\hline$C_{63,2} \perp$ & 56 & 1315 & 4725 & 54936 & 531048 & 4321791 & 30252537 & 184908360 & 998505144 & 4810848147 \\
\hline$C_{63,1 \perp}^{03,2 \perp}$ & 57 & 1651 & 9765 & 109368 & 1057224 & 8649279 & 60544953 & 369776680 & 1996794072 & 9621890019 \\
\hline
\end{tabular}

REMARK 8.1. Using the Jordan-Hölder Theorem for modules we deduce that the codes above are related as shown in the following proposition. We note that $C_{63,1}$ is a subcode of all these codes while $C_{63,1}{ }^{\perp}$ contains all of them. Some obvious and interesting properties of these codes can be deduced from their weight distributions. In Proposition 8.2 we show the containment of the codes and in Proposition 8.3, we collect their properties. 
TABLE 8 . Table 7 continued

\begin{tabular}{|c|c|c|c|c|c|c|}
\hline $\operatorname{dim}$ & 12 & 14 & 52 & 53 & 54 & 55 \\
\hline$C_{63,12 \perp}$ & 26208 & 216000 & $\ldots 6048$ & & & \\
\hline$C_{63,11 \perp}$ & 26208 & 216000 & . . 6048 & & & 945 \\
\hline$C_{63,10} \perp$ & 1421784 & 17856000 & . . 328104 & & & \\
\hline$C_{63,9 \perp}$ & 2697240 & 35668800 & . . 622440 & & 11200 & \\
\hline$C_{63,8} \perp$ & 1421784 & 17856000 & . . 328104 & 60480 & & 2016 \\
\hline$C_{63,7} \perp$ & 1421784 & 17856000 & . . 328104 & & & 9765 \\
\hline$C_{636 \perp}$ & 2697240 & 35668800 & $\ldots 622440$ & 60480 & 11200 & 11781 \\
\hline$C_{63,5} \perp$ & 20847008637 & 292087911600 & . . 4810848147 & & 184908360 & \\
\hline$C_{63,4} \perp$ & 41694856749 & 584173436400 & . . 9621890019 & & 369776680 & \\
\hline$C_{63,3} \perp$ & 20847008637 & 292087911600 & 4810848147 & 998288928 & 184908360 & 30292416 \\
\hline$C_{63,2 \perp}$ & 20847008637 & 292087911600 & 4810848147 & 998505144 & 184908360 & 30252537 \\
\hline$C_{63,1 \perp}$ & 41694856749 & 584173436400 & . . 9621890019 & 1996794072 & 369776680 & 60544953 \\
\hline
\end{tabular}

TABLE 9 . Table 7 continued

\begin{tabular}{|c|c|c|c|c|c|c|}
\hline $\operatorname{dim}$ & 56 & 57 & 58 & 59 & 60 & 63 \\
\hline$C_{63,12 \perp}$ & 135 & & & & & \\
\hline$C_{63,11 \perp}$ & 135 & & & & & 1 \\
\hline$C_{63,10 \perp}$ & 1395 & & & & & \\
\hline$C_{63,9 \perp}$ & 1683 & & & & & \\
\hline$C_{63,8^{\perp}}$ & 1395 & & & & & \\
\hline$C_{63,7} \perp$ & 1395 & & & & & 1 \\
\hline$C_{63,6} \perp$ & & & & & & 1 \\
\hline$C_{63,5^{\perp}}$ & 4321791 & & 54936 & & 315 & \\
\hline$C_{63,4} \perp$ & 8649279 & & 109368 & & 651 & \\
\hline$C_{63,3} \perp$ & 4321791 & 526176 & 54936 & 5040 & 315 & \\
\hline$C_{63,2} \perp$ & 4321791 & 531048 & 54936 & 4725 & 315 & 1 \\
\hline$C_{63,1} \perp$ & 8649279 & 1057224 & 109368 & 9765 & 651 & 1 \\
\hline
\end{tabular}

Proposition 8.2. $\quad$ (i) $C_{63,6} \subset C_{63,7}$;

(ii) $C_{63,6} \subset C_{63,8}$;

(iii) $C_{63,7}{ }^{\perp} \subset C_{63,6}{ }^{\perp}$;

(iv) $C_{63,1} \subset C_{63,2} \subset C_{63,5}$;

(v) $C_{63,1} \subset C_{63,6} \subset C_{63,9} \subset C_{63,10}$;

(vi) $C_{63,2} \subset C_{63,5}{ }^{\perp} \subset C_{63,3}{ }^{\perp} \subset C_{63,1}{ }^{\perp}$;

(vii) $C_{63,2} \subset C_{63,10}{ }^{\perp} \subset C_{63,7}{ }^{\perp} \subset C_{63,4}{ }^{\perp} \subset C_{63,1}{ }^{\perp}$;

(viii) $C_{63,1} \subset C_{63,3} \subset C_{63,5} \subset C_{63,10} \subset C_{63,12} \subset C_{63,11}{ }^{\perp} \subset C_{63,9}{ }^{\perp} \subset C_{63,1}{ }^{\perp}$.

Proposition 8.3. (i) The codes $C_{63, i}$ for $i \in\{4,5,9,10,12\}$ and $C_{63, i}{ }^{\perp}$ for $i \in\{2,6,7,11,12\}$ are self-complementary;

(ii) The codes $C_{63, i}$ for $i \in\{1,2,6,7,11\}$ are self-orthogonal and doublyeven. The codes $C_{63, i}{ }^{\perp}$ for $i \in\{5,6,12\}$ are singly even;

(iii) The codes $C_{63, i}$ where $i \in\{1,4\}$ and $C_{63, i}{ }^{\perp}$ for $i \in\{1,4,5,9,10\}$ are optimal, while $C_{63,5}$ and $C_{63, i}{ }^{\perp}$ for $i \in\{2,3,6,7\}$ are near-optimal;

(iv) Aut $_{63, i} \cong S_{6}(2)$ for $i \in\{2,3,5,6,8,9,11,12\}$, and $\mathrm{Aut} C_{63, i} \cong L_{6}(2)$ for $i \in\{1,4,7,10\}$. Moreover, $L_{6}(2)$ acts irreducibly on $C_{63,1}$ as an $\mathbb{F}_{2}$-module and for $i \in\{4,9,10,12\}$ the codes $C_{63, i}$ are decomposable. 
Proof. (i) That the codes are self-complementary follows readily since they contain the all-ones vector.

(ii) Arguing similarly as in the proof of Proposition 7.2 (ii), we observe that all but $C_{63,5}$, the codes are spanned by their minimum weight codewords. The block sizes of the designs supported by minimum-weight codewords in the codes $C_{63, i}$, where $i \in\{2,6,7,11\}$ have sizes $28,16,16$ and 12 respectively. Since these are all $\equiv 0(\bmod 4)$ we deduce that the corresponding codes are doubly even and hence self-orthogonal. In fact, for $i=1$ or 2 we have that $\operatorname{dim}\left(C_{63, i}\right)=\operatorname{dim} \operatorname{Hull}\left(C_{63, i}\right)$ which shows yet again that the two codes are self-orthogonal and doubly-even.

(iii) The optimality of the codes was verified computationally, however the reader can check that by consulting the online table of optimal codes $([16])$.

(iv) For the automorphism groups, we use the facts established in Proposition 8.2 as well as the following remarks. The codewords of weight 4 in $C_{63,12}$ span a subcode equivalent to $C_{63,11}$. Similarly, the codewords of weight 4 in $C_{63,10}$ span a subcode isomorphic to $C_{63,7}$ and $C_{63,6}$ is the code spanned by weight 4 codewords of $C_{63,9}$. Further we have $C_{63,9}=C_{63,6} \oplus\langle\mathbf{1}\rangle, C_{63,10}=C_{63,7} \oplus\langle\mathbf{1}\rangle$ and $C_{63,12}=C_{63,11} \oplus\langle\mathbf{1}\rangle$. Also $C_{63,5}=C_{63,3} \oplus\langle\mathbf{1}\rangle, C_{63,5}=C_{63,2} \oplus\langle\mathbf{1}\rangle, C_{63,4}=C_{63,1} \oplus\langle\mathbf{1}\rangle$, and $C_{63,10}=C_{63,8} \oplus\langle\mathbf{1}\rangle$. From this we deduce that $C_{63,4}, C_{63,5}, C_{63,9}, C_{63,10}$ and $C_{63,12}$ are all decomposable $\mathbb{F}_{2}$-modules. Now, if $\alpha \in \operatorname{Aut}\left(C_{63,6}\right)$ then since $\alpha(\mathbf{1})=\mathbf{1}$ and $C_{63,9}=C_{63,6} \oplus\langle\mathbf{1}\rangle$ we have $\alpha \in \operatorname{Aut}\left(C_{63,9}\right)$ and so $\operatorname{Aut}\left(C_{63,6}\right) \subseteq \operatorname{Aut}\left(C_{63,9}\right)$. Using the same argument we conclude that $\operatorname{Aut}\left(C_{63,1}\right) \subseteq \operatorname{Aut}\left(C_{63,4}\right), \operatorname{Aut}\left(C_{63,2}\right) \subseteq \operatorname{Aut}\left(C_{63,5}\right), \operatorname{Aut}\left(C_{63,11}\right) \subseteq$ $\operatorname{Aut}\left(C_{63,12}\right), \operatorname{Aut}\left(C_{63,7}\right) \subseteq \operatorname{Aut}\left(C_{63,10}\right)$ and $\operatorname{Aut}\left(C_{63,3}\right) \subseteq \operatorname{Aut}\left(C_{63,5}\right) . \quad \operatorname{By}$ computation with Magma we have $\left|\operatorname{Aut}\left(C_{63,3}\right)\right|=\left|\operatorname{Aut}\left(C_{63,5}\right)\right|=\left|\operatorname{Aut}\left(C_{63,2}\right)\right|$ $=1451520=\left|S_{6}(2)\right|$. Moreover, the support of the codewords of minimum weight 32 in $C_{63,1}$ yield a symmetric 2 - $(63,32,16)$ design which we denote $\mathfrak{D}$. Since $\operatorname{Aut}(\mathfrak{D}) \subseteq \operatorname{Aut}\left(C_{63,1}\right)$, and the complement $(\overline{\mathfrak{D}})$ of this design is a 2 - $(63,31,15)$ symmetric designs of points and hyperplanes of the projective geometry $P G(5,2)$, it follows that $\operatorname{Aut}(\mathfrak{D}) \cong P \Gamma L_{6}(2)$. Also, since $|\operatorname{Aut}(\mathfrak{D})|=20158709760=\left|\operatorname{Aut}\left(C_{63,1}\right)\right|$, we have $\operatorname{Aut}\left(C_{63,1}\right) \cong P \Gamma L_{6}(2)$. Now, the result follows using the earlier inclusions since for $i$ in each of the sets $\{1,4\},\{2,3,5\},\{6,9\},\{7,10\},\{11,12\}$ and $\{8\}$ the codes $\left(C_{63, i}\right)$ have isomorphic automorphism groups.

8.1. Stabilizer in $\operatorname{Aut}(C)$ of a word $w_{i}$ in a code $C$. In what follows we determine the structure of the stabilizers $(\operatorname{Aut}(C))_{w_{m}}$ where $m \in M$ where $M$ is defined as follows. Consider $M=\{27,28,31,32,35,36\}$ for the codes $C=C_{63, i}, 1 \leq i \leq 5$ and $M=\{15,48\}$ for the codes $C=C_{63, i}, 6 \leq i \leq 10$. For $m \in M$ we define $W_{m}=\left\{w_{m} \in C_{63, i} \mid \operatorname{wt}\left(w_{m}\right)=m\right\}$. In Lemma 8.4 we show that for all $m \in M$, the stabilizer $(\operatorname{Aut}(C))_{w_{m}}=H$ where $H<\operatorname{Aut}(C)$ is a maximal subgroup of $\operatorname{Aut}(C)$. In addition, for $w_{m} \in W_{m}$ we take the 
images of the support of $w_{m}$ under the action of $G=S_{6}(2)$ or $G=L_{6}(2)$ and form the blocks of the $t$ - $\left(63, m, k_{m}\right)(t \in\{1,2\})$ designs $\mathcal{D}=\mathcal{D}_{w_{m}}$, where $k_{m}=\left|\left(w_{m}\right)^{G}\right| \times \frac{m}{63}$ and show that $\operatorname{Aut}(C)$ acts primitively on $\mathcal{D}_{w_{m}}$. The information on these designs is given in Table 11.

Lemma 8.4. Let $C=C_{63, i}, 1 \leq i \leq 5$ be a code as in Proposition 8.3 and $0 \neq w_{m} \in C$. Then $\operatorname{Aut}(C)_{w_{m}}$ is a maximal subgroup of $\operatorname{Aut}(C)$. Moreover, the design $\mathcal{D}$ obtained by orbiting the images of the support of any non-trivial codeword in $C$ is primitive.

Proof. Clearly, from Proposition 8.3, the codes $C_{63, i}$ have either $S_{6}(2)$ or $L_{6}(2)$ as their automorphism group. We consider the action of these two groups on the codewords of weight $m \in M$ separately.

Case I: Let $\bar{G}=\operatorname{Aut}(C) \cong S_{6}(2)$, and $C=C_{63, i}$ where $i \in\{2,3,5\}$ and $M=\{27,28,31,32,35,36\}$. For all choices of $m \in M$, we have that $W_{m}$ is invariant under the action of $\bar{G}$ and from Table 5 and Table 6 we deduce that $W_{m}^{\bar{G}}=W_{m}$. Hence, each $W_{m}$ is a single orbit under this action, so that $\bar{G}$ acts transitively on $W_{m}$. Using the orbit stabilizer theorem we obtain $\left[\bar{G}: \bar{G}_{w_{m}}\right] \in\{28,36,63\}$. From the table of maximal subgroups of $S_{6}(2)$ (see Table 1) it can be deduced that $\left(S_{6}(2)\right)_{w_{m}} \in\left\{U_{4}(2): 2, S_{8}, 2^{5}: S_{6}\right\}$. Now, consider the codes $C_{63, i}$ for $i \in\{6,8,9\}$ and $M=\{15,48\}$. As above, we obtain that $W_{m}$ is invariant under $S_{6}(2)$ and $W_{m}^{\bar{G}}=W_{m}$ for all $m \in M$, and so $\bar{G}$ is transitive on each $W_{m}$. Moreover, $\left[\bar{G}_{:}: \bar{G}_{w_{m}}\right] \in\{315,336\}$. Thus we have $\left(S_{6}(2)\right)_{w_{m}} \in\left\{2 \cdot\left[2^{6}\right]: S_{3} \times S_{3}, S_{3} \times S_{6}\right\}$. Since $S_{6}(2)$ is transitive on the code coordinates, the support of the codewords of $W_{m}$ form the blocks of 1-designs $D_{w_{m}}$. The indices of $S_{6}(2)_{w_{m}}$ in $S_{6}(2)$ constitute the number of blocks of $D_{w_{m}}$. This implies that $S_{6}(2)$ is transitive on the blocks of $D_{w_{m}}$ for each $W_{m}$ and since $S_{6}(2)_{w_{m}}$ is a maximal subgroup of $S_{6}(2)$ for $m \in M$, we have that $S_{6}(2)$ acts primitively on $D_{w_{m}}$. The parameters of these designs are given in Table 11 and Table 12 respectively.

Case II. Let $\operatorname{Aut}(C) \cong L_{6}(2)$. In this case $C_{63, i}, i \in\{1,4\}$ and $M=$ $\{27,28,31,32,35,36\}$. For all choices of $m$ we have $\left(w_{m}\right)^{L_{6}(2)}=W_{m}$. Thus, $W_{m}$ is a single orbit of $L_{6}(2)$, and arguing similarly as in CASE I, we can show that $\left(L_{6}(2)\right)_{w_{m}}$ is a maximal subgroup of $L_{6}(2)$ isomorphic to $2^{5}: L_{5}(2)$. When $C_{63, i}, i \in\{7,10\}$ and $M=\{15,48\}$, we can show in a similar manner that $\left(L_{6}(2)\right)_{w_{m}}$ is a maximal subgroup of $L_{6}(2)$ isomorphic to $2^{7}:\left(S_{3} \times S_{3}\right)$. Hence, showing that $L_{6}(2)$ is primitive on the designs $D_{w_{m}}$.

REMARK 8.5. If we consider the action of $\operatorname{Aut}(C)$ on the codewords of the codes $C=C_{63, i}, i \in\{6,7,8\}$ of weight $m \in M$ with $M=$ $\{27,28,31,32,35,36\}$, it is found that $\operatorname{Aut}(C)$ splits $W_{m}$ into several orbits of different length where each may have a different subgroup as stabilizers. In some cases the stabilizer is maximal and in others it is not. For example Aut $\left(\mathrm{C}_{63,6}\right)$ acting on codewords of weight 32 splits these words into 9 orbits 
of length $181440,90720(2), 60840,15120,3780,6048,10080$ and 23040 and only acts primitively on the 9 -th orbit. This fact is indicated by writing $\left(32_{6}\right)_{9}$ in the Table 10 and Table 11. In Table 10 the first column gives the codes $C_{63, i}$, the second column represents the codewords of weight $m$ (the subindices $m$ represent the code from where the codeword is drawn), the third column gives the structure of the stabilizers in $\operatorname{Aut}(C)$ of a codeword $w_{m}$ and the last column, tests the maximality $(\operatorname{Aut}(C))_{w_{m}}$. In Table 11 the first column represents the codewords of weight $m$ and the second column gives the parameters of the $t$-designs $\mathcal{D}_{w_{m}}$ as defined in Section 8.1. In the third column we list the number of blocks of $\mathcal{D}_{w_{m}}$. The final column shows whether or not a design $\mathcal{D}_{w_{m}}$ is primitive under the action of $\operatorname{Aut}(C)$.

TABLE 10. Stabilizer in $\operatorname{Aut}(C)$ of a codeword $w_{m}$

\begin{tabular}{cccc||cccc}
\hline $\mathrm{C}$ & $m$ & $(\text { Aut }(\mathrm{C}))_{w_{m}}$ & Maximal & $\mathrm{C}$ & $m$ & $(\text { Aut }(\mathrm{C}))_{w_{m}}$ & Maximal \\
\hline \hline$C_{63,8}$ & $15_{8}$ & $S_{3} \times S_{6}$ & Yes & $C_{63,5}$ & $32_{5}$ & $2^{5}: S_{6}$ & Yes \\
$C_{63,9}$ & $15_{9}$ & $2 \cdot\left[2^{6}\right]:\left(S_{3} \times S_{3}\right)$ & Yes & $C_{63,6}$ & $\left(32_{6}\right)_{9}$ & $2^{5}: S_{6}$ & Yes \\
$C_{63,10}$ & $15_{10}$ & $2^{7}:\left(S_{3} \times S_{3}\right)$ & Yes & $C_{63,7}$ & $\left(32_{7}\right)_{3}$ & $2^{5}: L_{5}(2)$ & Yes \\
$C_{63,3}$ & $27_{3}$ & $U_{4}(2): 2$ & Yes & $C_{63,8}$ & $\left(32_{8}\right)_{9}$ & $2^{5}: S_{6}$ & Yes \\
$C_{63,5}$ & $27_{5}$ & $U_{4}(2): 2$ & Yes & $C_{63,9}\left(32_{9}\right)_{9}$ & $2^{5}: S_{6}$ & Yes \\
$C_{63,8}$ & $\left(27_{8}\right)_{6}$ & $U_{4}(2): 2$ & Yes & $C_{63,10}\left(32_{10}\right)_{3}$ & $2^{5}: L_{5}(2)$ & Yes \\
$C_{63,2}$ & $28_{2}$ & $S_{8}$ & Yes & $C_{63,3}$ & $35_{3}$ & $S_{8}$ & Yes \\
$C_{63,5}$ & $28_{5}$ & $S_{8}$ & Yes & $C_{63,5}$ & $35_{5}$ & $S_{8}$ & Yes \\
$C_{63,4}$ & $31_{4}$ & $2^{5}: L_{5}(2)$ & Yes & $C_{63,8}$ & $\left(35_{8}\right)_{6}$ & $S_{8}$ & Yes \\
$C_{63,5}$ & $31_{5}$ & $2^{5}: S_{6}$ & Yes & $C_{63,3}$ & $36_{2}$ & $U_{4}(2): 2$ & Yes \\
$C_{63,9}$ & $\left(31_{9}\right)_{9}$ & $2^{5}: S_{6}$ & Yes & $C_{63,5}$ & $36_{5}$ & $U_{4}(2): 2$ & Yes \\
$C_{63,10}\left(31_{10}\right)_{3}$ & $2^{5}: L_{5}(2)$ & Yes & $C_{63,6}$ & $48_{6}$ & $2 \cdot\left[2^{6}\right]:\left(S_{3} \times S_{3}\right)$ & Yes \\
$C_{63,1}$ & $32_{1}$ & $2^{5}: L_{5}(2)$ & Yes & $C_{63,7}$ & $48_{7}$ & $2^{7}:\left(S_{3} \times S_{3}\right)$ & Yes \\
$C_{63,2}$ & $32_{2}$ & $2^{5}: S_{6}$ & Yes & $C_{63,8}$ & $48_{8}$ & $2 \cdot\left[2^{6}\right]:\left(S_{3} \times S_{3}\right)$ & Yes \\
$C_{63,3}$ & $32_{3}$ & $2^{5}: S_{6}$ & Yes & $C_{63,9}$ & $48_{9}$ & $2 \cdot\left[2^{6}\right]:\left(S_{3} \times S_{3}\right)$ & Yes \\
$C_{63,4}$ & $32_{4}$ & $2^{5}: L_{5}(2)$ & Yes & $C_{63,10}$ & $48_{10}$ & $2^{7}:\left(S_{3} \times S_{3}\right)$ & Yes \\
\hline \hline
\end{tabular}

TABle 11. Primitive $t$-designs $\mathcal{D}_{w_{m}}$ invariant under $\operatorname{Aut}(C)$

\begin{tabular}{cccc||cccc}
\hline$m$ & $\mathcal{D}_{w_{m}}$ & No of blocks Prim & $m$ & $\mathcal{D}_{w_{m}}$ & \multicolumn{2}{c}{ No of blocks Prim } \\
\hline \hline $15_{8}$ & $1-(63,15,80)$ & 336 & Yes & $32_{5}$ & $1-(63,32,32)$ & 63 & Yes \\
$15_{9}$ & $1-(63,15,75)$ & 315 & Yes & $32_{6}$ & $1-(63,32,32)$ & 63 & Yes \\
$15_{10}$ & $2-(63,15,35)$ & 651 & Yes & 327 & $1-(63,32,32)$ & 63 & Yes \\
$27_{3}$ & $1-(63,27,12)$ & 28 & Yes & 328 & $1-(63,32,32)$ & 63 & Yes \\
$27_{5}$ & $1-(63,27,12)$ & 28 & Yes & 329 & $1-(63,32,32)$ & 63 & Yes \\
$27_{8}$ & $1-(63,27,12)$ & 28 & Yes & $32_{1} 10$ & $1-(63,32,32)$ & 63 & Yes \\
$28_{2}$ & $1-(63,28,16)$ & 36 & Yes & $35_{3}$ & $1-(63,35,20)$ & 36 & Yes \\
$28_{5}$ & $1-(63,28,16)$ & 36 & Yes & $35_{5}$ & $1-(63,35,20)$ & 36 & Yes \\
$31_{4}$ & $2-(63,31,31)$ & 63 & Yes & $35_{8}$ & $1-(63,35,20)$ & 36 & Yes \\
$31_{5}$ & $1-(63,31,31)$ & 63 & Yes & $36_{2}$ & $1-(63,36,16)$ & 28 & Yes \\
$31_{9}$ & $1-(63,31,31)$ & 63 & Yes & $36_{5}$ & $1-(63,36,16)$ & 28 & Yes \\
$31_{10}$ & $1-(63,31,31)$ & 63 & Yes & $48_{6}$ & $1-(63,48,240)$ & 315 & Yes \\
$32_{1}$ & $2-(63,32,32)$ & 63 & Yes & $48_{7}$ & $1-(63,48,96)$ & 651 & Yes \\
$32_{2}$ & $1-(63,32,32)$ & 63 & Yes & $48_{8}$ & $1-(63,48,240)$ & 315 & Yes \\
$32_{3}$ & $1-(63,32,32)$ & 63 & Yes & 489 & $1-(63,48,240)$ & 315 & Yes \\
$32_{4}$ & $2-(63,32,32)$ & 63 & Yes & $48_{10} 2-(63,48,376)$ & 651 & Yes \\
\hline
\end{tabular}

REMARK 8.6. (i) $\Gamma$ is a strongly regular $(63,32,16,16)$ graph. Since $\mu=\lambda=16$ we obtain a symmetric 2 - $(63,32,16)$ design, henceforth denoted 
by $\mathcal{D}$. The code $C_{63,1}$ of this design is a constant weight code, i.e., a code in which all non-zero codewords have same weight. The complement $\bar{\Gamma}$ is a strongly regular $(63,30,13,15)$ graph isomorphic to the symplectic graph $\mathcal{S}_{6}^{+}(2) . \quad \Gamma$ satisfies the triangle property and is uniquely determined by the minimality of its 2 -rank which is 6 . Notice though that $C_{63,1}$ code is the simplex code of dimension 6 and its dual $C_{63,1}^{\perp}$ is the Hamming code $H_{6}$, see $[3,18]$. The complement of $\mathcal{D}$ is the symmetric $2-(63,31,15)$ design $\overline{\mathcal{D}}$ of points and hyperplanes of the projective geometry $P G(5,2)$. $\mathcal{D}$ is also a Hadamard design and so extendible to a $3-(64,32,15)$ design $([8])$. The code of $\mathcal{D}$ is $C_{63,4}=C_{63,1} \oplus\langle\mathbf{1}\rangle$. These designs and codes are well known and their automorphism group is $L_{6}(2)$.

(ii) The words of minimum weight in $C_{63,1}^{\perp}$ can also be given a geometrical interpretation. The images under $\operatorname{Aut}\left(C_{63,4}\right)$ of the support of the codewords of minimum weight define a Steiner $2-(63,3,1)$ triple system which we denote $S T S(63)$. The 651 vectors of minimum weight generate $C_{63,1}^{\perp}$. Notice that $S T S(63)$ is the design of points and lines in $P G(5,2)$. It is a quasisymmetric design with block intersecting in 0 or 1 points. The block graph of this $S T S(63)$ is a strongly regular $(651,90,33,9)$ graph complemented by a strongly regular $(651,560,478,504)$ graph, see [25].

(iii) Lemma 8.4 can be of use in providing a geometric interpretation of the words of minimum weight in the codes examined. The words of weight 27 in $C_{63,3}$ and $C_{63,5}$ represent copies of $O_{6}^{-}(2)$ or the minus hyperplane in the orthogonal space. The stabilizer of any such codeword is a group isomorphic to $U_{4}(2): 2$. The words of weight 28 in $C_{63,2}$ and $C_{63,5}$ represent copies of an $O_{6}^{+}(2)$ or a plus hyperplane in the orthogonal space. The codewords of weight 31 in $C_{63,4}$ and $C_{63,5}$ and those of weight 32 for $C_{63, i}, i \in\{1,2,3,4,5\}$ represent the points of $\operatorname{PG}(5,2)$ or the isotropic points of the orthogonal space. They also represent the rows of the adjacency matrix of $\Gamma$ or equivalently the incidence vectors of the blocks of a $2-(63,31,15)$ symmetric design of points and hyperplanes of $P G(5,2)$. The set of codewords of weight 15 in $C_{63,8}, C_{63,9}$ and $C_{63,10}$ represent respectively the non-isotropic lines, the isotropic lines and the lines of $P G(5,2)$.

\section{The 120-DIMENSIONAL REPRESENTATION}

Notice that $S_{6}(2)$ acts primitively as a rank-3 group of degree 120 on the cosets of $U_{3}(3): 2$ with orbits of lengths 1,56 and 63 . This action defines a strongly regular $(120,56,28,24)$ graph. The complement of this graph is a strongly regular $(120,63,30,37)$ graph. These graphs are in the class of graphs partitioned by the symplectic graph and denoted $\mathcal{N}_{2 n}^{-}$where $n=4$, see [17]. The objects permuted in this action are copies of $G_{2}(2)$, see [12]. The permutation module splits into five absolutely irreducible constituents of dimensions 16,814 and 48 with multiplicities of 4, 4, 2, 
2 and 1 respectively. There are 2 irreducible submodules of dimension 1 and 8 both absolutely irreducible. Working through the chain of submodules of the permutation module we obtain in total 14 submodules of dimensions $119,112,111,105,91,85,84,36,35,29,15,9,8$ and 1 , and hence binary codes of these dimensions. The lattice of submodules is given in Figure 4 and the weight distributions of the codes are given in Table 12, Table 13 and Table 14.

FIGURE 4. Submodule lattice of the 120-dimensional representation

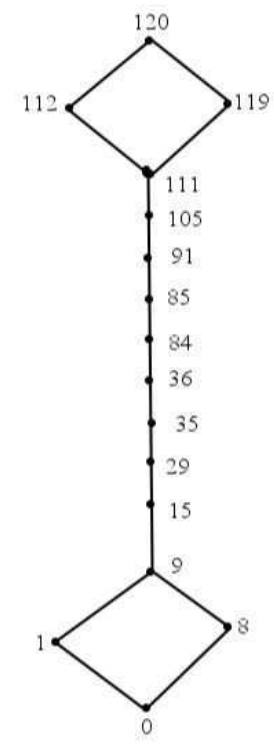

TABLE 12. The weight distribution of the codes from a 120dimensional representation.

\begin{tabular}{lllll}
\hline Name & $\operatorname{dim}$ & 0 & 56 & 64 \\
\hline \hline$C_{120,1}$ & 8 & 1 & 120 & 135 \\
\hline
\end{tabular}

TABLE 13. Table 12 continued.

\begin{tabular}{|c|c|c|c|c|c|c|c|c|c|}
\hline Name & $\operatorname{dim}$ & $\begin{array}{l}0 \\
120\end{array}$ & $\begin{array}{l}24 \\
96\end{array}$ & $\begin{array}{l}28 \\
92\end{array}$ & $\begin{array}{l}32 \\
88\end{array}$ & $\begin{array}{l}36 \\
84\end{array}$ & $\begin{array}{l}40 \\
80\end{array}$ & $\begin{array}{l}44 \\
76\end{array}$ & $\begin{array}{l}48 \\
72\end{array}$ \\
\hline$C_{120,2}$ & 9 & 1 & & & & & & & \\
\hline$C_{120,3}$ & 15 & 1 & & & & & 378 & & 630 \\
\hline$C_{120,4}$ & 29 & 1 & 315 & & 945 & 20160 & 119448 & 2459520 & 12133800 \\
\hline$C_{120,5}$ & 35 & 1 & 5355 & & 16065 & 1370880 & 8096760 & 145313280 & 884003400 \\
\hline$C_{120,6}$ & 36 & 1 & 5355 & 16320 & 16065 & 3089920 & 14676984 & 289255680 & 1794267720 \\
\hline
\end{tabular}


TABLE 14. Table 12 continued.

\begin{tabular}{l|llll}
\hline Name & dim & 52 & 56 & 60 \\
& & 68 & 64 & \\
\hline \hline$C_{120,2}$ & 9 & & 255 & \\
$C_{120,3}$ & 15 & & 15375 & \\
$C_{120,4}$ & 29 & 62233920 & 102040275 & 178854144 \\
$C_{120,5}$ & 35 & 3566142720 & 7413648915 & 10322543616 \\
$C_{120,6}$ & 36 & 7041968640 & 14999707155 & 20433469056 \\
\hline
\end{tabular}

Proposition 9.1. (i) The code $C_{120,1}$ is a $[120,8,56]_{2}$ self-orthogonal, doubly-even and projective code. Its dual $C_{120,1}{ }^{\perp}$ is a $[120,112,3]_{2}$ singly even and uniformly packed. $C_{120,1}{ }^{\perp}$ is a near-optimal code. Moreover, $\operatorname{Aut}\left(C_{120,1}\right) \cong O_{8}^{+}(2): 2$ which acts irreducibly on $C_{120,1}$.

(ii) $C_{120,2}$ is a self-orthogonal and doubly-even. It is a $[120,9,56]_{2}$ code, and its dual $C_{120,2}{ }^{\perp}$ is a $[120,111,4]_{2}$ singly even code. Moreover, $C_{120,2}$ and $C_{120,2}{ }^{\perp}$ are optimal codes and $\operatorname{Aut}\left(C_{120,2}\right) \cong S_{8}(2)$.

(iii) $C_{120,3}$ is a $[120,15,40]_{2}$ self-orthogonal, doubly-even and decomposable code. Its dual $C_{120,3}{ }^{\perp}$ is a $[120,105,4]_{2}$ singly even code and $\operatorname{Aut}\left(C_{120,3}\right) \cong S_{6}(2)$.

(iv) $C_{120,4}$ is a self-orthogonal and doubly-even $[120,29,24]_{2}$ code. Its dual $C_{120,4}{ }^{\perp}$ is a $[120,91,8]_{2}$ singly even code. $\mathbf{1} \in C_{120,4}{ }^{\perp}$ and $\operatorname{Aut}\left(C_{120,4}\right) \cong S_{6}(2)$.

(v) $C_{120,5}$ is a self-orthogonal, doubly-even code. It is a $[120,35,24]_{2}$ code, and its dual $C_{120,5}{ }^{\perp}$ is a $[120,85,8]_{2}$ singly even code. Also, $\mathbf{1} \in C_{120,5}$ and $\mathbf{1} \in C_{120,5}{ }^{\perp}$ and $\operatorname{Aut}\left(C_{120,5}\right) \cong S_{8}(2)$.

(vi) $C_{120,6}=[120,36,24]_{2}$ is a self-orthogonal and doubly-even code. Its dual $C_{120,6}{ }^{\perp}$ is a $[120,84,8]_{2}$ singly even code. $\mathbf{1} \in C_{120,6}$ and $\mathbf{1} \in$ $C_{120,6}{ }^{\perp}$ and $\operatorname{Aut}\left(C_{120,6}\right) \cong S_{8}(2)$.

Proof. The proof follows the same arguments as those used in the previous propositions, so we omit the details.

REMARK 9.2. (i) The words of weight 56 in $C_{120,1}$ have a geometrical interpretation. They represent the rows of the adjacency matrix of the graph $\Gamma=(120,56,28,24)$ or equivalently the incidence vectors of the blocks of the symmetric $2-(120,56,56)$ design. The code $C_{120,1}=[120,8,56]$ is part of a family of known codes of type $\left[2^{2 m-1}-2^{m-1}, 2 m+1,2^{2 m-2}-2^{m-1}\right]$.

(ii) Notice that $C_{120,1}$ is a two-weight code. It follows from [7] that this code defines a strongly regular $(256,120,56,56)$ graph $\Lambda$ complemented by a strongly regular $(256,135,70,72)$ graph $\bar{\Lambda}$. Since $\lambda=\mu$ we have that $\Lambda$ is in fact a symmetric $2-(256,56,56)$ design.

(iii) The words of weight 56 in $C_{120,1}$ represent copies of $G_{2}(2)$. These words are stabilized by a group isomorphic to $U_{3}(3)$. The set of codewords of weight 24 in $C_{120,4}$ represent the isotropic lines and the stabilizer of an isotropic line is a group isomorphic to $2 \cdot\left[2^{6}\right]:\left(S_{3} \times S_{3}\right)$. Since $\operatorname{Aut}\left(C_{120,1}\right)=$ 
$O_{8}^{+}(2): 2$ and $\operatorname{Aut}\left(C_{120,4}\right)=S_{6}(2)$, we deduce that $O_{8}^{+}(2): 2$ acts primitively on isomorphic copies of $G_{2}(2)$, and $S_{6}(2)$ acts primitively on the set of isotropic lines. The set of codewords of weight 64 in $C_{120,1}$ represent isotropic planes. The stabilizer of an isotropic plane is isomorphic to $2^{6}: L_{3}(2)$. Hence $O_{8}^{+}(2): 2$ acts primitively on the isotropic planes.

(iv) The code $C_{120,6}$ has 5355 codewords of minimum weight. The supports of the 5355 minimum words form the blocks of a $2-(120,24,207)$ design $\mathcal{D}_{120,6}$ for which $\operatorname{Aut}\left(\mathcal{D}_{120,6}\right)=\operatorname{Aut}\left(C_{120,5}+\mathbf{1}\right)$, and this group is isomorphic to the simple symplectic group $S_{8}(2)$. The automorphism group is 2-transitive on points, primitive on blocks, so the 5355 minimum weight codewords are in one orbit under $\operatorname{Aut}\left(C_{120,5}+\mathbf{1}\right)$. Moreover, the all-one vector $\mathbf{1}$ is the sum of all the rows. Observe that both $C_{120,5}{ }^{\perp}$ and $C_{120,6}{ }^{\perp}$ have minimum weight 8 . The fact that the minimum weight of $C_{120,6}{ }^{\perp}$ is at least 8 follows also from the design parameters, since the replication number $r$ for $\mathcal{D}_{120,6}$ is 1071 . This design and corresponding codes were also constructed in [15] using an adjacency matrix for the uniform subset graph $\Gamma(10,3,0)$, i.e. 3 -sets from a set of size 10 with adjacency if the sets do not intersect.

By determining all $S_{6}(2)$-invariant submodules, the distinct codes of length 120 respectively are known, and a result we conclude that there is not self-dual $S_{6}(2)$-invariant binary code of this length. Thus we have

Proposition 9.3. Up to isomorphism there are exactly 12 non-trivial codes of length 120 invariant under $S_{6}(2)$. Moreover, there is no self-dual code of length 120 invariant under $S_{6}(2)$

\section{THE 135-DIMENSIONAL REPRESENTATION}

$S_{6}(2)$ acts as rank-4 group primitive permutation group of degree 135 on the cosets of $2^{6}: L_{3}(2)$ with orbits of lengths $1,14,56$ and 64 . Using this action we form a permutation module of dimension 135 invariant under $G$. The elements being permuted in this action are isotropic planes. The permutation module splits into 5 absolutely irreducible constituents of dimension 1, 6, 8, 14 and 48 with multiplicities of 5, 5, 3, 2 and 1 respectively. There are two irreducible submodules of dimensions 1 and 8 , both absolutely irreducible. Working as in the earlier permutation representations we obtain submodules of dimensions $1,8,9,14,15(3), 16,28,29(2), 34,35(10), 36(13), 37(3), 41,42(7)$, $43(15), 44(8), 45,49,50(6), 51(7), 52$ and their duals. The digits in brackets represent the number of modules of the corresponding length. Due to computer time limitations we are unable to determine all submodules and hence codes of length 135 invariant under $S_{6}(2)$. As a result, in Figure 5 we give a partial lattice diagram. We were able to enumerate a total of 172 nontrivial binary codes of length 135. A summary of the properties of the codes found is given in Table 15. 
Figure 5. Partial submodule lattice of the 135-dimensional representation

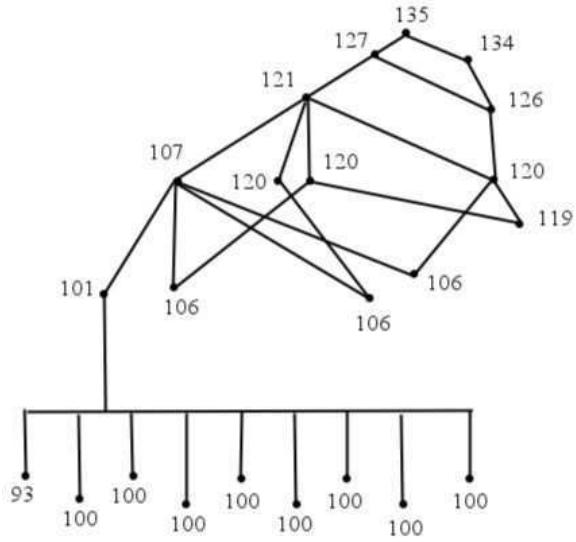

Table 15: Properties of codes from the 135-dimensional representation.

\begin{tabular}{|c|c|c|c|c|c|c|c|c|c|c|c|c|c|}
\hline Name & code & wds & Aut & S.O & s.e. & d.e. & 1 & opt & dual & wds & s.e & d.e & 1 \\
\hline$C_{135,1}$ & {$[135,8,64]$} & 135 & $\mathrm{O}_{8}^{+}(2): 2$ & $\bar{t}$ & $t$ & $t$ & $f$ & $f$ & {$[135,127,3]$} & 1575 & $\bar{f}$ & $f$ & $t$ \\
\hline$C_{135,2}$ & {$[135,9,63]$} & 120 & $O_{8}^{+}(2): 2$ & $f$ & $f$ & $f$ & & $f$ & {$[135,126,4]$} & 5195 & $t$ & $f$ & $f$ \\
\hline$C_{135,3}$ & {$[135,14,48]$} & 630 & $S_{6}(2)$ & $t$ & $t$ & $t$ & & $f$ & {$[135,121,3]$} & 315 & $f$ & $f$ & $t$ \\
\hline$C_{135,4}$ & {$[135,15,48]$} & 630 & $S_{6}(2)$ & $f$ & $f$ & $f$ & $t$ & $f$ & {$[135,120,4]$} & 2835 & $f$ & $f$ & $t$ \\
\hline$C_{135,5}$ & {$[135,15,30]$} & 36 & $S_{6}(2)$ & $t$ & $t$ & $f$ & $f$ & $f$ & {$[135,120,3]$} & 315 & $f$ & $f$ & $t$ \\
\hline$C_{135,6}$ & {$[135,15,48]$} & 630 & $S_{6}(2)$ & $f$ & $f$ & $f$ & $t$ & $f$ & {$[135,120,4]$} & 2835 & $t$ & $f$ & $f$ \\
\hline$C_{135,7}$ & {$[135,16,30]$} & 360 & $S_{6}(2)$ & $f$ & $f$ & $f$ & & $f$ & {$[135,119,4]$} & 2835 & $t$ & $f$ & $f$ \\
\hline$C_{135,8}$ & {$[135,28,32]$} & 95 & $S_{6}(2)$ & $t$ & $t$ & $t$ & $f$ & $f$ & {$[135,107,5]$} & 378 & $f$ & $f$ & $t$ \\
\hline$C_{135,9}$ & {$[135,29,30]$} & 36 & $S_{6}(2)$ & $t$ & $t$ & $f$ & $f$ & $f$ & {$[135,106,5]$} & 378 & $f$ & $f$ & $t$ \\
\hline$C_{135,10}$ & {$[135,29,32]$} & 945 & $S_{6}(2)$ & $f$ & $f$ & $f$ & $t$ & $f$ & {$[135,106,6]$} & 630 & $f$ & $f$ & $t$ \\
\hline$C_{135,11}$ & {$[135,30,30]$} & 36 & $S_{6}(2)$ & $f$ & $f$ & $f$ & $t$ & $f$ & {$[135,105,6]$} & 630 & $t$ & $f$ & $f$ \\
\hline$C_{135,12}$ & {$[135,34,32]$} & 12285 & $O_{8}^{+}(2): 2$ & & $t$ & $t$ & $f$ & $f$ & {$[135,101,7]$} & 12285 & $f$ & $f$ & $t$ \\
\hline$C_{135,13}$ & {$[135,35,31]$} & 3780 & $O_{8}^{+}(2): 2$ & $f$ & $f$ & $f$ & $t$ & $f$ & {$[135,100,8]$} & 32400 & $t$ & $f$ & $f$ \\
\hline$C_{135,14}$ & {$[135,35,30]$} & 36 & $S_{6}(2)$ & $t$ & $t$ & $f$ & $f$ & $f$ & {$[135,100,7]$} & 945 & $f$ & $f$ & $t$ \\
\hline$C_{135,15}$ & {$[135,35,24]$} & 945 & $S_{6}(2)$ & $f$ & $t$ & $f$ & $f$ & $f$ & {$[135,100,6]$} & 630 & $f$ & $f$ & $t$ \\
\hline$C_{135,16}$ & {$[135,35,24]$} & 1260 & $S_{6}(2)$ & $f$ & $t$ & $f$ & $f$ & $f$ & {$[135,100,5]$} & 378 & $f$ & $f$ & $t$ \\
\hline$C_{135,17}$ & {$[135,35,32]$} & 12285 & $S_{6}(2)$ & $f$ & $f$ & $f$ & $f$ & $f$ & {$[135,100,7]$} & 1080 & $f$ & $f$ & $f$ \\
\hline$C_{135,18}$ & {$[135,35,31]$} & 3780 & $\mathrm{O}_{8}^{+}(2): 2$ & $f$ & $f$ & $f$ & $f$ & $f$ & {$[135,100,8]$} & 32400 & $t$ & $f$ & $f$ \\
\hline$C_{135,19}$ & {$[135,35,32]$} & 12285 & $S_{6}(2)$ & $f$ & $f$ & $f$ & $f$ & $f$ & {$[135,100,7]$} & 1080 & $f$ & $f$ & $f$ \\
\hline$C_{135,20}$ & {$[135,35,30]$} & 36 & $S_{6}(2)$ & $t$ & $t$ & $f$ & $f$ & $f$ & {$[135,100,7]$} & 945 & $f$ & $f$ & $t$ \\
\hline$C_{135,21}$ & {$[135,35,32]$} & 12285 & $S_{6}(2)$ & $t$ & $t$ & $f$ & $f$ & $f$ & {$[135,100,7]$} & 945 & $f$ & $f$ & $t$ \\
\hline$C_{135,22}$ & {$[135,35,28]$} & 4320 & $\mathrm{O}_{8}^{+}(2): 2$ & $t$ & $t$ & $t$ & $f$ & $f$ & {$[135,100,7]$} & 2025 & $f$ & $f$ & $t$ \\
\hline$C_{135,23}$ & {$[135,36,30]$} & 36 & $S_{6}(2)$ & $f$ & $f$ & $f$ & $t$ & $f$ & {$[135,99,8]$} & 16200 & $t$ & $f$ & $f$ \\
\hline$C_{135,24}$ & {$[135,36,24]$} & 1260 & $S_{6}(2)$ & $f$ & $f$ & $f$ & & $f$ & {$[135,99,8]$} & 13365 & $t$ & $f$ & $f$ \\
\hline$C_{135,25}$ & {$[135,36,15]$} & 63 & $S_{6}(2)$ & $f$ & $f$ & $f$ & & $f$ & {$[135,99,6]$} & 630 & $t$ & $f$ & $f$ \\
\hline$C_{135,26}$ & {$[135,36,27]$} & 1120 & $O_{8}^{+}(2): 2$ & $f$ & $f$ & $f$ & $t$ & $f$ & {$[135,99,8]$} & 32400 & $t$ & $f$ & $f$ \\
\hline$C_{135,27}$ & {$[135,36,27]$} & 1120 & $S_{6}(2)$ & $f$ & $f$ & $f$ & $f$ & $f$ & {$[135,99,8]$} & 16200 & $f$ & $f$ & $f$ \\
\hline$C_{135,28}$ & {$[135,36,27]$} & 1120 & $S_{6}(2)$ & $f$ & $f$ & $f$ & $f$ & $f$ & {$[135,99,8]$} & 16200 & $f$ & $f$ & $f$ \\
\hline$C_{135,29}$ & {$[135,36,28]$} & 4320 & $S_{6}(2)$ & $t$ & $t$ & $f$ & $f$ & $f$ & {$[135,99,7]$} & 945 & $f$ & $f$ & $t$ \\
\hline$C_{135,30}$ & {$[135,36,24]$} & 945 & $S_{6}(2)$ & $f$ & $f$ & $f$ & $f$ & $f$ & {$[135,99,8]$} & 13365 & $f$ & $f$ & $f$ \\
\hline$C_{135,31}$ & {$[135,36,24]$} & 945 & $S_{6}(2)$ & $f$ & $t$ & $f$ & $f$ & $f$ & {$[135,99,8]$} & 13365 & $f$ & $f$ & $t$ \\
\hline$C_{135,32}$ & {$[135,36,24]$} & 1260 & $S_{6}(2)$ & $f$ & $t$ & $f$ & $f$ & $f$ & {$[135,99,8]$} & 13365 & $t$ & $f$ & $t$ \\
\hline
\end{tabular}


Table 15 - continued

\begin{tabular}{|c|c|c|c|c|c|c|c|c|c|c|c|c|c|}
\hline Name & code & wds & Aut & S.O & s.e & d.e & 1 & opt & dual & wds & s.e & d.e & 1 \\
\hline$C_{135,33}$ & {$[135,36,24]$} & 1260 & $S_{6}(2)$ & $\bar{f}$ & $\bar{f}$ & $\bar{f}$ & $\bar{t}$ & $\bar{f}$ & {$[135,99,8$} & 13365 & $\bar{f}$ & $\bar{f}$ & $f$ \\
\hline$C_{135,34}$ & {$[135,36,15]$} & 72 & $S_{6}(2)$ & $f$ & $f$ & $f$ & $f$ & $f$ & {$[135,99,5]$} & 378 & $f$ & $f$ & $f$ \\
\hline$C_{135,35}$ & {$[135,36,28]$} & 4320 & $S_{6}(2)$ & $f$ & $f$ & $f$ & $f$ & $f$ & {$[135,99,7]$} & 1080 & $t$ & $f$ & $f$ \\
\hline$C_{135,36}$ & {$[135,37,15]$} & 63 & $S_{6}(2)$ & $f$ & $f$ & $f$ & $t$ & $f$ & {$[135,98,8]$} & 13365 & $t$ & $f$ & $f$ \\
\hline$C_{135,37}$ & {$[135,37,15]$} & 72 & $S_{6}(2)$ & $f$ & $f$ & $f$ & $t$ & $f$ & {$[135,98,8]$} & 13365 & $t$ & $f$ & $f$ \\
\hline$C_{135,38}$ & {$[135,37,27]$} & 1120 & $S_{6}(2)$ & $f$ & $f$ & $f$ & $t$ & $f$ & {$[135,98,8]$} & 16200 & $t$ & $f$ & $f$ \\
\hline$C_{135,39}$ & {$[135,41,24]$} & 2205 & $S_{6}(2)$ & $f$ & $t$ & $f$ & $f$ & $f$ & {$[135,94,8]$} & 2025 & $f$ & $f$ & $t$ \\
\hline$C_{135,40}$ & {$[135,42,24]$} & 4725 & $O_{8}^{+}(2)$ & $f$ & $t$ & $f$ & $t$ & $f$ & {$[135,93,8]$} & 2025 & $f$ & $f$ & $t$ \\
\hline$C_{135,41}$ & {$[135,42,15]$} & 63 & $S_{6}(2)$ & $f$ & $f$ & $f$ & $t$ & $f$ & {$[135,93,8]$} & 2025 & $t$ & $f$ & $f$ \\
\hline$C_{135,42}$ & {$[135,42,24]$} & 2025 & $S_{6}(2)$ & $f$ & $f$ & $f$ & $f$ & $f$ & {$[135,93,8]$} & 2025 & $f$ & $f$ & $f$ \\
\hline$C_{135,43}$ & {$[135,42,24]$} & 2025 & $S_{6}(2)$ & $f$ & $t$ & $f$ & $f$ & $f$ & {$[135,93,8]$} & 2025 & $f$ & $f$ & $t$ \\
\hline$C_{135,44}$ & {$[135,42,24]$} & 2025 & $S_{6}(2)$ & $f$ & $t$ & $f$ & $f$ & $f$ & {$[135,93,8$} & 2025 & $f$ & $f$ & $t$ \\
\hline$C_{135,45}$ & {$[135,42,15]$} & 72 & $S_{6}(2)$ & $f$ & $f$ & $f$ & $t$ & $f$ & {$[135,91,8]$} & 2025 & $f$ & $f$ & $f$ \\
\hline$C_{135,46}$ & {$[135,42,24]$} & 4725 & $O_{8}^{+}(2)$ & $f$ & $t$ & $f$ & $f$ & $f$ & {$[135,93,8]$} & 945 & $f$ & $f$ & $t$ \\
\hline$C_{135,47}$ & {$[135,43,24]$} & 4725 & $O_{8}^{+}(2)$ & $t$ & $f$ & $f$ & $f$ & $f$ & {$[135,92,8]$} & 2025 & $f$ & $f$ & $t$ \\
\hline$C_{135,48}$ & {$[135,43,24]$} & 4725 & $S_{6}(2)$ & $f$ & $f$ & $f$ & $f$ & $f$ & {$[135,92,8]$} & 945 & $f$ & $f$ & $f$ \\
\hline$C_{135,49}$ & {$[135,43,15]$} & 135 & $O_{8}^{+}(2)$ & $f$ & $f$ & $f$ & $t$ & $f$ & {$[135,92,8]$} & 2025 & $t$ & $f$ & $f$ \\
\hline$C_{135,50}$ & {$[135,43,24]$} & 4725 & $S_{6}(2)$ & $t$ & $f$ & $f$ & $f$ & $f$ & {$[135,92,8]$} & 945 & $f$ & $f$ & $t$ \\
\hline$C_{135,51}$ & {$[135,43,24]$} & 4725 & $O_{8}^{+}(2)$ & $f$ & $f$ & $f$ & $f$ & $f$ & {$[135,92,8]$} & 2025 & $f$ & $f$ & $f$ \\
\hline$C_{135,52}$ & {$[135,43,24]$} & 4725 & $S_{6}(2)$ & $f$ & $f$ & $f$ & $t$ & $f$ & {$[135,92,8]$} & 945 & $f$ & $f$ & $t$ \\
\hline$C_{135,53}$ & {$[135,43,24]$} & & $S_{6}(2)$ & $t$ & $f$ & $f$ & $f$ & $f$ & {$[135,92,8]$} & & $f$ & $f$ & $t$ \\
\hline$C_{135,54}$ & {$[135,43,24]$} & & $S_{6}(2)$ & $t$ & $f$ & $f$ & $f$ & $f$ & {$[135,92,8]$} & & $f$ & $f$ & $t$ \\
\hline$C_{135,55}$ & {$[135,43,15]$} & & $O_{8}^{+}(2)$ & $f$ & $f$ & $f$ & $t$ & $f$ & {$[135,92,8]$} & & $f$ & $f$ & $f$ \\
\hline$C_{135,56}$ & {$[135,43,15]$} & & $S_{6}(2)$ & $f$ & $f$ & $f$ & $t$ & $f$ & {$[135,92,8]$} & & $t$ & $f$ & $f$ \\
\hline$C_{135,57}$ & {$[135,43,24]$} & & $O_{8}^{+}(2)$ & $f$ & $f$ & $f$ & $f$ & $f$ & {$[135,92,8]$} & & $t$ & $f$ & $f$ \\
\hline$C_{135,58}$ & {$[135,43,15]$} & & $S_{6}(2)$ & $f$ & $f$ & $f$ & $f$ & $f$ & {$[135,92,8]$} & & $f$ & $f$ & $f$ \\
\hline$C_{135,59}$ & {$[135,43,24]$} & & $O_{8}^{+}(2)$ & $t$ & $f$ & $f$ & $f$ & $f$ & {$[135,92,8]$} & & $f$ & $f$ & $t$ \\
\hline$C_{135,60}$ & {$[135,43,24]$} & & $O_{8}^{+}(2)$ & $t$ & $f$ & $f$ & $f$ & $f$ & {$[135,92,8]$} & & $f$ & $f$ & $t$ \\
\hline$C_{135,61}$ & {$[135,43,24]$} & & $S_{6}(2)$ & $f$ & $f$ & & $f$ & $f$ & {$[135,92,8$} & & $f$ & $f$ & $t$ \\
\hline$C_{135,62}$ & {$[135,44,15]$} & & $S_{6}(2)$ & $f$ & $f$ & $f$ & $t$ & $f$ & {$[135,91,8]$} & & $t$ & $f$ & $f$ \\
\hline$C_{135,63}$ & {$[135,44,24]$} & & $S_{6}(2)$ & $f$ & $f$ & $f$ & $f$ & $f$ & {$[135,91,8]$} & & $f$ & $f$ & $f$ \\
\hline$C_{135,64}$ & {$[135,51,24]$} & & & $f$ & $f$ & $f$ & $t$ & $f$ & {$[135,91,8]$} & & $t$ & $f$ & $f$ \\
\hline$C_{135,65}$ & {$[135,44,24]$} & & $S_{6}(2)$ & $f$ & $t$ & $f$ & $f$ & $f$ & {$[135,91,8]$} & & $f$ & $f$ & $f$ \\
\hline$C_{135,66}$ & {$[135,44,24]$} & & $S_{6}(2)$ & $f$ & $f$ & $f$ & $f$ & $f$ & {$[135,91,8]$} & & $f$ & $f$ & $t$ \\
\hline$C_{135,67}$ & {$[135,44,15]$} & & $S_{6}(2)$ & $f$ & $f$ & $f$ & $f$ & $f$ & {$[135,91,8]$} & & $f$ & $f$ & $f$ \\
\hline$C_{135,68}$ & {$[135,44,15]$} & & $S_{6}(2)$ & $f$ & $f$ & $f$ & & $f$ & {$[135,91,8]$} & & $t$ & $f$ & $f$ \\
\hline$C_{135,69}$ & {$[135,44,15]$} & & $O_{8}^{+}(2)$ & $f$ & $f$ & $f$ & & $f$ & {$[135,91,8]$} & & $t$ & $f$ & $f$ \\
\hline$C_{135,70}$ & {$[135,45,15]$} & & $S_{6}^{\circ}(2)$ & $f$ & $f$ & $f$ & & $f$ & {$[135,90,8]$} & & $t$ & $f$ & $f$ \\
\hline$C_{135,71}$ & {$[135,49,16]$} & & $S_{6}(2)$ & $f$ & $t$ & $f$ & $f$ & $f$ & {$[135,86,9]$} & & $f$ & $f$ & $t$ \\
\hline$C_{135,72}$ & {$[135,50,15]$} & & $S_{6}(2)$ & $f$ & $f$ & $f$ & $t$ & $f$ & {$[135,85]$,} & & $t$ & $f$ & $f$ \\
\hline$C_{135,73}$ & {$[135,50,16]$} & & $S_{6}(2)$ & $f$ & $f$ & $f$ & $f$ & $f$ & {$[135,85]$,} & & $f$ & $f$ & $f$ \\
\hline$C_{135,74}$ & {$[135,43,16]$} & & $O_{8}^{+}(2)$ & $f$ & $t$ & $f$ & & $f$ & {$[135,85$,} & & $f$ & $f$ & $t$ \\
\hline$C_{135,76}$ & {$[135,50,15]$} & & $S_{6}(2)$ & $f$ & $f$ & $f$ & $f$ & $f$ & {$[135,85$,} & & $f$ & $f$ & $f$ \\
\hline$C_{135,77}$ & {$[135,50,16]$} & & & $f$ & $f$ & $f$ & $f$ & $f$ & {$[135,85$,} & & $f$ & $f$ & $f$ \\
\hline$C_{135,78}$ & {$[135,50,16]$} & & & $f$ & $t$ & $f$ & $f$ & $f$ & {$[135,85$,} & & $f$ & $f$ & $t$ \\
\hline$C_{135,79}$ & {$[135,51,15]$} & & & $f$ & $f$ & $f$ & & $f$ & {$[135,84$,} & & $t$ & $f$ & $f$ \\
\hline$C_{135,80}$ & {$[135,51,15]$} & & & $f$ & $f$ & $f$ & & $f$ & {$[135,84$,} & & $t$ & $f$ & $f$ \\
\hline$C_{135,81}$ & {$[135,51,15]$} & & & $f$ & $f$ & $f$ & & $f$ & {$[135,84$,} & & $t$ & $f$ & $f$ \\
\hline$C_{135,82}$ & {$[135,51,16]$} & & & $f$ & $t$ & $f$ & $f$ & $f$ & {$[135,84$,} & & $f$ & $f$ & $f$ \\
\hline$C_{135,83}$ & {$[135,51,15]$} & & & $f$ & $f$ & $f$ & $f$ & $f$ & {$[135,84$,} & & $f$ & $f$ & $f$ \\
\hline$C_{135,84}$ & {$[135,51,15]$} & & & $f$ & $f$ & $f$ & $f$ & $f$ & {$[135,84$,} & & $f$ & $f$ & $f$ \\
\hline$C_{135,85}$ & {$[135,51,15]$} & & & $f$ & $f$ & $f$ & $f$ & & {$[135,84$,} & & $f$ & $f$ & $t$ \\
\hline & {$[135,52,15]$} & & & $f$ & $f$ & $J$ & & $f$ & {$[135,83]$,} & & $t$ & & $f$ \\
\hline
\end{tabular}

In Table 15, the first column gives a label for the code, the second gives the parameters of the code, the third gives the number of codewords of a given weight where possible, the fourth column gives the structure of the automorphism group. From the fifth to the ninth columns we have true 
("t") indicating if the code is self-orthogonal (s.o.), singly-even (s.e.), doubly even (d.e.), presence of the all-one vector (1) in the code or optimality, and false ("f") otherwise. The last column deals with the properties of the dual code. We have computed the weight distributions up to dimension 44 . Currently Magma is unable to give the computations for higher dimensions. Consequently the relations between the codes could not be fully established. A summary of the codes found and their properties is given in Tables 16 and 17 .

TABLE 16. Weight distribution of codes of length 135.

\begin{tabular}{|c|c|c|c|c|c|c|c|c|c|c|c|c|c|}
\hline $\operatorname{dim}$ & $\begin{array}{lll}015 \\
\end{array}$ & 24 & 27 & 28 & 29 & 30 & 31 & 32 & 33 & 34 & 35 & 36 & 37 \\
\hline$\overline{8}$ & 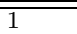 & & & & & & & & & & & & \\
\hline 9 & 1 & & & & & & & & & & & & \\
\hline 14 & 1 & & & & & & & & & & & & \\
\hline 15 & 1 & & & & & & & & & & & & \\
\hline 15 & 1 & & & & & 36 & & & & & & & \\
\hline 15 & 1 & & & & & & & & & & & & \\
\hline 16 & 1 & & & & & 36 & & & & & & & \\
\hline 28 & 1 & & & & & & & 945 & & & & 3360 & \\
\hline 29 & 1 & & & & & 36 & & 945 & & & & 3360 & \\
\hline 29 & 1 & & & & & & & 945 & & & & 3360 & \\
\hline 35 & 1 & & & & & & 3780 & 12285 & & & 12096 & 33600 & \\
\hline 35 & 1 & & & & & 36 & & 12285 & & & & 33600 & \\
\hline 35 & 1 & 945 & & & & 1044 & & 945 & & & & 43680 & \\
\hline 35 & 1 & 1260 & & & & 1296 & & 4725 & & & & 33600 & \\
\hline 35 & 1 & & & & & & & 12285 & 1260 & & & 33600 & \\
\hline 35 & 1 & & & & & & 3780 & 12285 & & & 12096 & 33600 & \\
\hline 35 & 1 & & & & & & & 12285 & & & & 33600 & 4320 \\
\hline 35 & 1 & & & & & 36 & & 12285 & & & & 33600 & \\
\hline 35 & 1 & & & & & & & 12285 & & & & 33600 & \\
\hline 35 & 1 & & & 4320 & & & & 12285 & & & & 45600 & \\
\hline 36 & 1 & & & & & 36 & 3780 & 12285 & 1260 & & 12096 & 33600 & \\
\hline 36 & 1 & 1260 & & & & 1296 & & 4725 & & & & 33600 & \\
\hline 36 & 163 & 945 & & & & 1044 & 3780 & 945 & 16380 & & & 43680 & \\
\hline 36 & 1 & & 1120 & 4320 & & & 3780 & 12285 & & & 16416 & 45600 & \\
\hline 36 & 1 & & 1120 & & & 36 & & 12285 & & & 4320 & 33600 & 4320 \\
\hline 36 & 1 & & 1120 & & & & & 12285 & 1260 & & 4320 & 33600 & \\
\hline 36 & 1 & & & 4320 & & 36 & & 12285 & & & & 45600 & \\
\hline 36 & 1 & 945 & & & & 1044 & & 945 & & & 8640 & 43680 & \\
\hline 36 & 1 & 945 & & & & 1044 & & 945 & & & & 52320 & \\
\hline 36 & 1 & 1260 & & & & 1296 & & 4725 & & & & 33600 & \\
\hline 36 & 172 & 1260 & & & & 1296 & 7560 & 4725 & 20160 & & & 33600 & \\
\hline 36 & 1 & & & 4320 & & & & 12285 & 1260 & & & 45600 & 4320 \\
\hline 36 & 1 & & & & & & 3780 & 12285 & & & 12096 & 33600 & 4320 \\
\hline 42 & 1 & 4725 & & & & 4320 & & 68985 & & & & 638400 & \\
\hline 42 & 163 & 2205 & & & & 2304 & 30240 & 38745 & 80640 & & 12096 & 315840 & \\
\hline 42 & 1 & 2205 & 1120 & & & 2304 & & 38745 & & & 276480 & 315840 & 276480 \\
\hline 42 & 1 & 2205 & & 4320 & & 2304 & & 38745 & & & & 882240 & \\
\hline 42 & 1 & 2205 & & & & 2304 & & 38745 & & & & 949440 & \\
\hline 42 & 1 & 4725 & & & & 4320 & & 68985 & & & & 638400 & \\
\hline 42 & 172 & 2205 & & & & 2304 & 30240 & 38745 & 70560 & & & 315840 & \\
\hline 44 & 1135 & 4725 & & 4320 & 1080 & 8136 & 60480 & 68985 & 194040 & 128520 & 163296 & 977760 & 430920 \\
\hline
\end{tabular}


TABLE 17 . Table 16 continued

\begin{tabular}{|c|c|c|c|c|c|c|c|c|c|c|c|}
\hline $\operatorname{dim}$ & $\ldots 101$ & 102 & 103 & 104 & 105 & 106 & 107 & 108 & 111 & 120 & 135 \\
\hline$\overline{88}$ & $\ldots$ & & & & & & & & & & \\
\hline 9 & $\ldots$ & & & & & & & & & & 1 \\
\hline 14 & $\ldots$ & & & & & & & & & & \\
\hline 15 & $\ldots$ & & & & 36 & & & & & & \\
\hline 15 & $\ldots$ & & & & & & & & & & \\
\hline 15 & $\ldots$ & & & & & & & & & & 1 \\
\hline 16 & $\ldots$ & & & & 36 & & & & & & 1 \\
\hline 28 & $\ldots$ & & & & & & & & & & \\
\hline 29 & $\ldots$ & & & & & & & & & & \\
\hline 29 & $\ldots$ & & & & 36 & & & & & & \\
\hline 35 & $\ldots$ & & 12285 & 3780 & & & & & & & 1 \\
\hline 35 & $\ldots$ & 1260 & & 3780 & & & & & & & \\
\hline 35 & $\ldots$ & 16380 & & 3780 & & & & & & 63 & \\
\hline 35 & $\ldots$ & & & & & & & & & & \\
\hline 35 & $\ldots$ & & & 3780 & 36 & & & & & & \\
\hline 35 & $\ldots$ & & & 3780 & & & & & & & \\
\hline 35 & $\ldots$ & & & 3780 & & & & & & & \\
\hline 35 & $\ldots$ & & & 3780 & & & & 1120 & & & \\
\hline 36 & $\ldots$ & 1260 & 12285 & 3780 & 36 & & & & & & 1 \\
\hline 36 & $\ldots$ & & 4725 & & 1296 & & & & 1260 & & 1 \\
\hline 36 & $\ldots$ & 16380 & 945 & 3780 & 1044 & & & & 945 & 63 & 1 \\
\hline 36 & $\ldots$ & & 12285 & 3780 & & & 4320 & 1120 & & & 1 \\
\hline 36 & $\ldots$ & 1260 & & 3780 & & & 4320 & & & & \\
\hline 36 & $\ldots$ & & & 3780 & 36 & & 4320 & & & & \\
\hline 36 & $\ldots$ & 1260 & & 3780 & & & & 1120 & & & \\
\hline 36 & $\ldots$ & 16380 & & 3780 & & & & & & 63 & \\
\hline 36 & $\ldots$ & 16380 & & 3780 & & & & & & 63 & \\
\hline 36 & $\ldots$ & 20160 & & 7560 & & & & & & 72 & \\
\hline 36 & $\ldots$ & & & & & & & & & & \\
\hline 36 & $\ldots$ & & & 3780 & 36 & & & 1120 & & & \\
\hline 36 & $\ldots$ & & 12285 & 3780 & & & & & & & 1 \\
\hline 42 & $\ldots$ & 151200 & & 60480 & & & & & & 135 & \\
\hline 42 & $\ldots$ & 80640 & 38745 & 30240 & 2304 & & & & 2205 & 63 & 1 \\
\hline 42 & $\ldots$ & 80640 & & 30240 & & & 4320 & & & 63 & \\
\hline 42 & $\ldots$ & 80640 & & 30240 & & & & 1120 & & 63 & \\
\hline 42 & $\ldots$ & 80640 & & 30240 & & & & & & 63 & \\
\hline 42 & $\ldots$ & 151200 & & 60480 & & & & & & 135 & \\
\hline 42 & $\ldots$ & 80640 & 30240 & 30240 & 2016 & & & & 2520 & 63 & \\
\hline 44 & . . 128520 & 194040 & 68985 & 60480 & 8136 & 1080 & 4320 & & 4725 & 135 & 1 \\
\hline
\end{tabular}

\section{REFERENCES}

1. Atlas of finite group representations - Version 3, http://brauer.maths.qmul.ac.uk/ Atlas/clas/S62/.

2. J. L. Alperin and R. B. Bell, Groups and representations, Springer-Verlag, New York Inc., 1995.

3. E. F. Assmus, Jr and J. D. Key, Designs and their codes, Cambridge University Press, Cambridge, 1992.

4. W. Bosma, J. Cannon and C. Playoust, The Magma algebra system. I. The user language, J. Symbolic Comput. 24 (1997), 235-265.

5. P. L. H. Brooke, On matrix representations and codes associated with the simple group of order 25920, J. Algebra 91 (1984), 536-566.

6. P. L. H. Brooke, On the Steiner systems $S(2,4,28)$ and codes associated with the simple group of order 6048. J. Algebra 97 (1985), 376-406. 
7. R. Calderbank and W. M. Kantor, The geometry of two-weight codes, Bull. London Math. Soc. 18 (1986), 97-122.

8. P. J. Cameron and J. H. van Lint, Designs, graphs, codes and their links, Cambridge University Press, Cambridge, 1991.

9. L. Chikamai, Linear codes obtained from 2-modular representations of some finite simple groups, PhD thesis, University of KwaZulu-Natal, 2013.

10. L. Chikamai, J. Moori and B. G. Rodrigues, Some 2-modular codes preserved by the simple group $L_{3}(4)$, Util. Math. 95 (2014), 357-399.

11. L. Chikamai, J. Moori and B. G. Rodrigues, 2-modular representations of the alternating group $A_{8}$ as binary codes, Glas. Mat. Ser. III. 47(67) (2012), 225-252.

12. J. H. Conway, R. T. Curtis, S. P. Norton, R. A. Parker and R. A. Wilson, Atlas of finite groups, Oxford University Press, Oxford, 1985.

13. D. Crnković and V. Mikulić Crnković, On some combinatorial structures constructed from the groups $L(3,5), U(5,2)$ and $S(6,2)$. Int. J. Comb. 237 (2011), Article ID 137356, 12 pages.

14. S. M. Dodunekov, S. B. Encheva and S. N. Kapralov, On the $[28,7,12]$ binary selfcomplementary codes and their residuals, Des. Codes Cryptogr. 4 (1994), 57-67.

15. W. Fish, J. D. Key and E. Mwambene, Binary codes from reflexive graphs on 3-set, submitted.

16. M. Grassl, Bounds on the minimum distance of linear codes and quantum codes, online available at http://www. codetables.de, 2007. Accessed on 05-08-2013.

17. W. H. Haemers, R. Peeters, and J. M. van Rijckevorsel, Binary codes of strongly regular graphs, Des. Codes Cryptogr. 17 (1999), 187-209.

18. R. Hill, A first course in coding theory, Oxford University Press, Oxford, 1986.

19. C. Jansen, K. Lux, R. Parker and R. Wilson, An atlas of Brauer characters, Oxford Scientific Publications, Clarendon Press, Oxford, 1995.

20. J. D. Key and J. Moori, Codes, designs and graphs from the Janko groups $J_{1}$ and $J_{2}$, J. Combin. Math. Combin. Comput. 40 (2002), 143-159.

21. C. Parker, E. Spence and V. D. Tonchev, Designs with the symmetric difference property on 64 points and their groups, J. Combin. Theory Ser. A 67 (1994), 23-43.

22. R. Peeters, Uniqueness of strongly regular graphs having minimal p-rank, Linear Algebra Appl. 226/228 (1995), 9-31.

23. J. Rotman, An introduction to the theory of groups, Springer-Verlag, New York, 1995.

24. D. Seiple, Investigation of binary self-dual codes invariant under simple groups, MSc thesis, The University of Arizona, 2009.

25. M. S. Shrikhande and S. S. Sane, Quasi-symmetric designs, Cambridge University Press, Cambridge, 1991.

26. R. A. Wilson, The finite simple groups, Springer-Verlag London Ltd., London, 2009. 
L. Chikamai

School of Mathematics, Statistics and Computer Science

University of KwaZulu-Natal

Durban 4000

South Africa

E-mail: lucychikamai@gmail.com

J. Moori

School of Mathematical Sciences

North-West University (Mafikeng)

Mmabatho 2735

South Africa

E-mail: Jamshid.Moori@nwu.ac.za

B. G. Rodrigues

School of Mathematics, Statistics and Computer Science

University of KwaZulu-Natal

Durban 4000

South Africa

E-mail: rodrigues@ukzn.ac.za

Received: 23.8.2013.

Revised: 3.12.2013. 